\title{
Equilibrium Tropical Cyclone Size in an Idealized State of Axisymmetric Radiative-Convective Equilibrium*
}

\author{
DANIEl R. CHAVAS AND KERRY EMANUEL \\ Massachusetts Institute of Technology, Cambridge, Massachusetts
}

(Manuscript received 29 May 2013, in final form 3 January 2014)

\begin{abstract}
Tropical cyclone size remains an unsolved problem in tropical meteorology, yet size plays a significant role in modulating damage. This work employs the Bryan cloud model (CM1) to systematically explore the sensitivity of the structure of an axisymmetric tropical cyclone at statistical equilibrium to the set of relevant model, initial, and environmental external parameters. The analysis is performed in a highly idealized state of radiative-convective equilibrium (RCE) governed by only four thermodynamic parameters, which are shown to modulate the storm structure primarily via modulation of the potential intensity.

Using dimensional analysis, the authors find that the equilibrium radial wind profile is primarily a function of a single nondimensional parameter given by the ratio of the storm radial length scale to the parameterized eddy radial length scale. The former is found to be the ratio of the potential intensity to the Coriolis parameter, matching the prediction for the "natural" storm length scale embedded within prevailing axisymmetric tropical cyclone theory; the Rossby deformation radius is shown not to be fundamental. Beyond this primary scaling, a second nondimensional parameter representing the nondimensional Ekman suction velocity is found to modulate the far outer wind field. Implications of the primary nondimensional parameter are discussed, including the critical role of effective turbulence in modulating inner-core structure and new insight into empirical estimates of the radial mixing length.
\end{abstract}

\section{Introduction}

Considerable progress has been made over the past three decades in elucidating the dynamics of tropical cyclones (TCs). Theory has been developed suggesting that TCs may be viewed as a Carnot heat engine whose heat source arises from the ambient thermodynamic disequilibrium of the tropical oceans (Emanuel 1986). Furthermore, both theory and relatively simple dynamical models (Ooyama 1969; DeMaria and Pickle 1988; Rotunno and Emanuel 1987; Emanuel 1995a) are able to reproduce many of the characteristic features of mature tropical cyclones, including maximum wind speed, central sea level pressure, and thermodynamic structure.

\footnotetext{
* Supplemental information related to this paper is available at the Journals Online website: http://dx.doi.org/10.1175/JAS-D-130155.s1.

Corresponding author address: Daniel R. Chavas, Massachusetts Institute of Technology, 77 Massachusetts Ave. 54-1715, Cambridge, MA 02139.

E-mail: drchavas@gmail.com
}

Most recently, Emanuel and Rotunno (2011) derived a full analytical solution for the radial structure of the axisymmetric balanced tropical cyclone wind field at the top of the boundary layer.

However, this latest solution remains defined relative to a single free parameter: the outer radius $r_{0}$, where the winds vanish. Indeed, despite wide recognition of the sensitivity of both storm surge (Irish et al. 2008) and wind damage (Iman et al. 2005) to storm size, size remains largely unpredictable. In the absence of interaction with land or extratropical disturbances, size is observed in nature to vary significantly more from storm to storm than within the lifetime of a given storm, regardless of basin, location, and time of year (Merrill 1984; Frank 1977; Chavas and Emanuel 2010; Lee et al. 2010). Size is found to correlate only weakly with both latitude and intensity (Merrill 1984; Weatherford and Gray 1988; Chavas and Emanuel 2010), as the outer- and inner-core regions appear to evolve nearly independently. Chavas and Emanuel (2010) found that the global distribution of $r_{0}$ is approximately lognormal, though distinct median sizes exist within each ocean basin, suggesting that the size of a given TC is not merely a global random variable 
but instead is likely modulated either by the structure of the initial disturbance, the environment in which it is embedded, or both. Idealized modeling studies in Hill and Lackmann (2009) and Xu and Wang (2010) found that TCs tend to be larger when embedded in moister midtropospheric environments. Using simple three-layer axisymmetric models, DeMaria and Pickle (1988) found size at peak intensity increased with increasing background rotation rate, while Smith et al. (2011) found an optimum in storm size. Finally, Rotunno and Emanuel (1987) demonstrated a strong relationship between the horizontal length scales of the initial and mature vortex.

This work seeks to build on the small base of literature on tropical cyclone size by systematically exploring the sensitivity of the structure of an axisymmetric tropical cyclone at statistical equilibrium to the set of relevant model, initial, and environmental external parameters. Expanding on the work of Hakim (2011), we perform our analysis in the simplest possible model and physical environment: a highly idealized state of radiativeconvective equilibrium (RCE). The results of the sensitivity analysis are then synthesized via dimensional analysis to quantify the relationship between equilibrium storm structure and the set of relevant input parameters. Section 2 details the methodology and experimental design, including derivation of a useful alternative formulation of the maximum potential intensity in the context of our idealized RCE environment. Results and accompanying discussion are presented in section 3. Finally, section 4 provides a brief summary and conclusions.

\section{Methodology}

\section{a. Model description}

We construct a highly idealized model and environmental configuration to explore equilibrium tropical cyclone structure in RCE using version 15 of the Bryan cloud model (CM1; Bryan and Fritsch 2002), which incorporates state-of-the-art numerics and physics, in particular for moist processes, while satisfying near-exact conservation of both mass and energy in a reversible saturated environment. The model can conveniently be run with nearly identical parameters in three-dimensional or axisymmetric (radius-height) geometry.

CM1 solves the fully compressible equations of motion in height coordinates on an $f$ plane for flow velocities $(u, v, w)$, nondimensional pressure $\pi$, potential temperature $\theta$, and the mixing ratios of water in vapor, liquid, and solid states $q_{\chi}$ on a fully staggered Arakawa C-type grid in height coordinates. The model has a rigid lid at the top with a 5-km-thick damping layer beneath and a wall at the domain's outer horizontal edge with an adjacent damping layer whose thickness is set to approximately $1 / 15$ of the domain's width. The damping time scale is set to its default value of $6 \mathrm{~min}$. Model horizontal $(x-y)$ and vertical grid spacing are each constant in the domain. Model microphysics is represented using the GoddardLin-Farley-Orville (LFO) scheme based on Lin et al. (1983), which is a mixed-phase bulk ice scheme with prognostic equations for water vapor, cloud water, rainwater, pristine ice crystals, snow, and large ice. For full details, see Bryan and Fritsch (2002). In lieu of a comprehensive scheme for radiative transfer, an idealized scheme (discussed below) is imposed because of its simplicity. Finally, turbulence is parameterized using a Smagorinsky-type closure scheme (Smagorinsky 1963), which assumes steady and homogeneous unresolved turbulence, modified such that different eddy viscosities are used for the horizontal/radial and vertical directions to represent the differing nature of turbulence between the radial and vertical directions in a highly anisotropic system, such as in the inner core of a tropical cyclone. In the context of tropical cyclones, turbulence fulfills the critical role of counteracting eyewall frontogenesis by the secondary circulation that, in the inviscid limit, would lead to frontal collapse (Emanuel 1997).

\section{b. Idealized model/environmental RCE setup}

We construct a highly idealized model and environmental configuration with the objective of reducing the model atmospheric system to the simplest possible state (i.e., minimal number of dimensional variables) that supports a tropical cyclone. Model horizontal and vertical grid spacings are set to $d x=d y=d r=4 \mathrm{~km}$ and $d z=0.625 \mathrm{~km}$, respectively, and no grid stretching is applied. Surface pressure is set to $1015 \mathrm{hPa}$. Radiation is represented simply by imposing a constant cooling rate (which is typical of the clear-sky mean tropical troposphere; see Hartmann et al. 2001) $Q_{\text {cool }}$ to the potential temperature everywhere in the domain where the absolute temperature exceeds a threshold value $T_{\text {tpp }}$; below this value, Newtonian relaxation back to this threshold is applied:

$$
\frac{\partial \theta}{\partial t}= \begin{cases}-Q_{\mathrm{cool}} & T>T_{\mathrm{tpp}} \\ \frac{\theta\left(p, T_{\mathrm{tpp}}\right)-\theta(p, T)}{\tau} & T \leq T_{\mathrm{tpp}}\end{cases}
$$

where $T$ is absolute temperature, and $\tau$ is the relaxation time scale, set to 40 days. Thus, all water-radiation and temperature-radiation feedbacks are neglected. The sea surface temperature $T_{\text {sst }}$ is set constant. Surface fluxes of enthalpy and momentum are calculated using standard bulk aerodynamic formulas 


$$
\begin{aligned}
& F_{k}=C_{k} \rho|\mathbf{u}|\left(k_{s}^{*}-k\right), \\
& \tau_{s}=-C_{d} \rho|\mathbf{u}| \mathbf{u},
\end{aligned}
$$

where $F_{k}$ is the surface enthalpy flux, $\rho$ is the near-surface air density, $\mathbf{u}$ is the near-surface (i.e., lowest model level) wind velocity, $k$ is the near-surface enthalpy, $k_{s}^{*}$ is the saturation enthalpy of the sea surface, $\tau_{s}$ is the surface stress, and the exchange coefficients for momentum $C_{d}$ and enthalpy $C_{k}$ are set constant, despite their acknowledged real-world dependence on wind speed (Powell et al. 2003). Finally, background surface enthalpy fluxes are required to balance column radiative cooling in order to achieve RCE in the absence of significant resolved wind perturbations (such as a tropical cyclone). Because axisymmetric geometry precludes the direct imposition of a background flow, we instead simply add a constant gustiness $u_{\text {sfc }}$ to $|\mathbf{u}|$ for the model calculation of (2) and (3). This setup is conceptually similar to that of Hakim (2011) with the important exceptions that here we employ a noninteractive radiative scheme and we include background surface fluxes throughout the domain.

This configuration provides a simplified framework for the exploration of equilibrium tropical cyclone structure in RCE. Nolan et al. (2007) demonstrated that, in the presence of a full radiation scheme, the $f$-plane RCE state depends only on $T_{\mathrm{sst}}, u_{\mathrm{sfc}}$, and very weakly on the Coriolis parameter $f$. For this work, the idealized radiation scheme introduces two additional degrees of freedom, $T_{\text {tpp }}$ and $Q_{\text {cool }}$, to which the RCE state is sensitive. Thus, we initialize each axisymmetric simulation with the RCE solution from the corresponding threedimensional simulation on a $196 \times 196 \mathrm{~km}^{2}$ domain with identical $T_{\text {st }}, T_{\text {tpp }}, Q_{\text {cool }}$, and $u_{\text {sfc }}$; the RCE state is indeed found to be nearly insensitive to $f$ (not shown) and thus it is held constant at its control value to reduce computational load. This domain size is specifically chosen to be large enough to permit a large number of updrafts but small enough to inhibit convective self-aggregation (Bretherton et al. 2005) over a period of at least 100 days. The RCE solution is defined as the 30-day time- and horizontal-mean vertical profiles of potential temperature and water vapor, with the threshold for equilibrium defined as $\partial \theta / \partial t<1 / 30 \mathrm{~K} \mathrm{day}^{-1}$ over the equilibrium period at all model levels. Overall, this approach ensures that each axisymmetric simulation begins very close to its "natural" model-equilibrated 3D RCE background state (Rotunno and Emanuel 1987) and thus is absent any significant stores of available potential energy that may exist by imposing an alternate initial state, such as a mean tropical sounding.

The result of the above methodology is a model RCE atmosphere comprised of a troposphere capped by a nearly isothermal stratosphere at $T_{\mathrm{tpp}}$. More generally, this model tropical atmosphere may be thought of as an extension of the classical fluid system in which a fluid is heated from below and cooled from above (albeit throughout the column), but with two key modifications: 1) the energy input into the system is dependent on wind speed, thereby permitting a wind-induced surface heat exchange (WISHE; Emanuel 1986) feedback, and 2) the energy lost from the system is dependent on an externally defined temperature threshold $\left(T_{\mathrm{tpp}}\right)$, which conveniently corresponds to the convective outflow temperature central to the maximum potential intensity theory of tropical cyclones. Both modifications facilitate a more straightforward methodology and analysis of the factors that modulate equilibrium storm size and structure.

\section{c. Initial perturbation}

Bister and Emanuel (1997) demonstrated that the fundamental process during tropical cyclogenesis is the near saturation of the column at the mesoscale in the core of the nascent storm. Thus, we superpose an initial perturbation upon the background RCE state by saturating the air at constant virtual temperature in a region above the boundary layer bounded by $z=[1.5,9.375] \mathrm{km}$ and $r=\left(0, r_{0_{q}}\right)$ within a quiescent environment. We also test an initial midlevel vortex of the form used in Rotunno and Emanuel (1987), characterized by a radius of vanishing wind $r_{0_{u}}$ and a peak wind of $V_{m_{0}}=12.5 \mathrm{~m} \mathrm{~s}^{-1}$ at $r_{m_{0}}=r_{0_{u}} / 5$, centered at $z=4.375 \mathrm{~km}$ with azimuthal wind speeds above and below decaying linearly to zero over a distance of $2.875 \mathrm{~km}$. However, the two approaches have similar results (see Fig. 6), and thus for the sake of simplicity, we elect to initialize all other simulations with the midlevel moisture anomaly. In addition to this initial disturbance, random perturbations with magnitudes uniformly distributed in the range $[-1,1] \mathrm{K}$ are added to the potential temperature field at every point to break the initial horizontal symmetry of the model.

\section{d. Control simulation parameter values}

For the control simulation, values of the key external parameters for the model, environmental, and initial condition are provided in Table 1 . The values of the horizontal and vertical mixing lengths $\left(l_{h}\right.$ and $l_{v}$, respectively), used in the Smagorinsky-type parameterization of three-dimensional turbulence are typical values taken from the literature (Bryan and Rotunno 2009a). The corresponding initial three-dimensional RCE vertical profile of potential temperature and water vapor is displayed in Fig. 1.

The domain size for the control run requires special attention. Prior research modeling tropical cyclones typically place the outer wall of the domain at a distance 
TABLE 1. Parameter values for the control simulation. See text for details.

\begin{tabular}{llllr}
\hline \hline Model & & & & \multicolumn{1}{c}{ Initial } \\
condition & Value & $r_{0_{q}}$ & Value \\
\hline$l_{h}$ & Value & Environment & $300 \mathrm{~K}$ & $200 \mathrm{~km}$ \\
$l_{v}$ & $1500 \mathrm{~m}$ & $T_{\text {sst }}$ & $200 \mathrm{~K}$ & $400 \mathrm{~km}$ \\
$C_{k}, C_{d}$ & $100 \mathrm{~m}$ & $T_{\text {tpp }}$ & $1 \mathrm{~K} \mathrm{day}^{-1}$ & \\
$H_{\text {domain }}$ & 0.0015 & $Q_{\text {cool }}$ & $3 \mathrm{~m} \mathrm{~s}^{-1}$ & \\
$L_{\text {domain }}$ & $25 \mathrm{~km}$ & $u_{\text {sfc }}$ & $5 \times 10^{-5} \mathrm{~s}^{-1}$ & \\
\hline
\end{tabular}

of 1000-1500 km (e.g., Rotunno and Emanuel 1987; Hakim 2011). However, as shown in Fig. 2, which depicts the day-100-150 mean radial profile of the azimuthal component of the gradient wind at $z=1.56 \mathrm{~km}$, storm size is dramatically influenced by the radius of the outer wall up to an upper bound. Beyond this upper bound, the equilibrium storm is largely insensitive to the location of the wall. Thus, because the outer wall is purely a model artifact, we set the outer wall conservatively at $L_{\text {domain }}=$ $12288 \mathrm{~km}$ for all simulations run herein, which also ensures that the storm itself does not significantly alter the environment.

\section{e. Characterizing equilibrium storm structure}

All simulations are run for 150 days in order to allow sufficient time for the full tropical cyclone structure to reach statistical equilibrium, and data are output at 6-h intervals. We then calculate a 2-day running mean of the

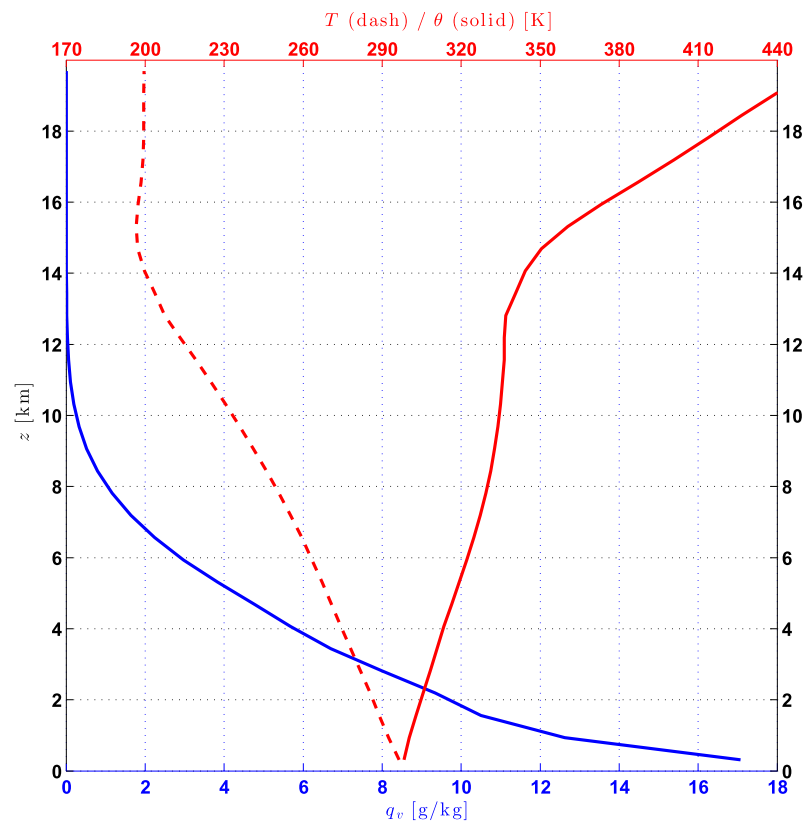

FIG. 1. Initial three-dimensional radiative-convective equilibrium vertical profile of temperature (red dashed), potential temperature (red solid), and water vapor mixing ratio (blue) for the control simulation. radial profile of the azimuthal gradient wind at $z=$ $1.56 \mathrm{~km}$ to reduce noise in the pressure field. Given that extant tropical cyclone structure and potential intensity theory applies strictly to the gradient wind, we choose the gradient rather than full wind for radial wind profiles; the role of supergradient winds is discussed in, for example, Smith and Montgomery (2008), Smith and Vogl (2008), and Rotunno and Bryan (2012). Results are not sensitive to the output frequency or the averaging period length. We calculate the gradient wind directly from model prognostic variables based on gradient wind balance:

$$
V_{g}=-\frac{1}{2} f r+\left(\frac{1}{4} f^{2} r^{2}+r C_{p} \theta_{v} \frac{\partial \pi}{\partial r}\right)^{1 / 2}
$$

The equilibrium radial wind profile is defined as the time mean of the 30-day period after day 60 with the minimum time variance in $V_{m}$ to account for simulations that exhibit significant long-period variability in storm structure.

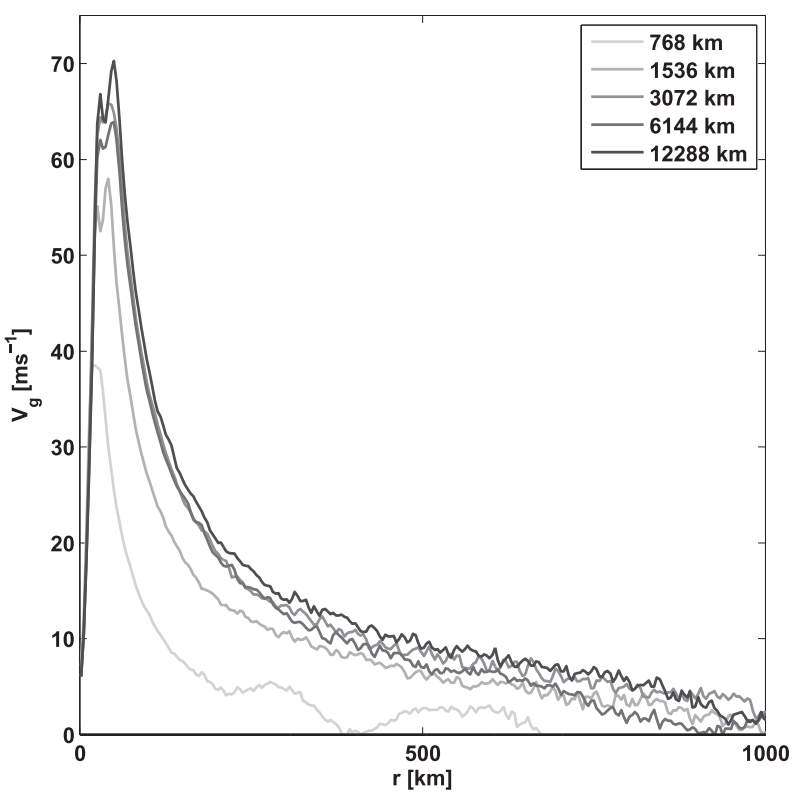

FIG. 2. Time-mean radial gradient wind profiles at $z=1.56 \mathrm{~km}$ for days $100-150$ as a function of domain width. Note the convergence in storm size beyond $L_{\text {domain }} \approx 3000 \mathrm{~km}$. 
For two cases $\left(f=10^{-4} \mathrm{~s}^{-1}, l_{h}=750 \mathrm{~m}\right)$, the equilibrium period was adjusted manually to account for ongoing structural variability. Though simplistic, this definition provides a clean signal in many of the details discussed below.

Following the theory presented in Emanuel and Rotunno (2011), we would ideally characterize the structure of the tropical cyclone wind field near the top of the boundary layer with three variables: the maximum gradient wind speed $V_{m}$, the radius of maximum gradient wind $r_{m}$, and the outer radius $r_{0}$, which corresponds to the first point moving outwards from the center where the wind goes to zero. However, variability in the structure of the gradient wind in the eyewall renders the precise $r_{m}$ noisy. In particular, as was found in Bryan and Rotunno (2009a), despite a single local maximum in the full wind, in most cases there exist two local maxima in the gradient wind, located at the inner and outer edges of the eyewall. This behavior may be an artifact of the use of the full $\pi$ for calculating $V_{g}$ in the eyewall where supergradient winds exist; the pitfalls of this approach are discussed in Bryan and Rotunno (2009a). The peak gradient wind is typically found at the outer maximum, but cases where it is found at the inner radius, even if only temporarily, can significantly bias the estimate of $r_{m}$. Thus, as a proxy, we will track the radius of $75 \%$ of $V_{m}$ outside of the eyewall $r_{\mathrm{ew}}$, which is more stable and typically scales closely with $r_{m}$.

Meanwhile, direct calculation of $r_{0}$ is problematic because of the larger variability toward the outer edge of the model storm $\left(V \lesssim 0.1 V_{m}\right)$ and correspondingly large sensitivity of the precise value of $r_{0}$ to this variability. Instead, we employ the outer wind structure model derived in Emanuel (2004) to estimate $r_{0}$. This model assumes that the flow is steady, axisymmetric, and absent deep convection, resulting in a local balance between subsidence warming and radiative cooling. The equilibriumsubsidence velocity $w_{\text {cool }}$ can be taken to be approximately constant with radius for a given background RCE state. In equilibrium, this subsidence rate must match the rate of Ekman suction-induced entrainment of free-tropospheric air into the boundary layer in order to prevent the creation of large vertical temperature gradients across the top of the boundary layer. The radial profile of azimuthal velocity is therefore determined as that which provides the required Ekman suction and is governed by the following differential equation

$$
\frac{\partial(r V)}{\partial r}=\frac{2 r^{2} C_{d} V^{2}}{w_{\text {cool }}\left(r_{0}^{2}-r^{2}\right)}-f r,
$$

where $r$ is the radius and $V$ is the azimuthal wind speed. Equation (5) is a Riccati equation with no known analytical solution but can be solved using a shooting method. The value of $w_{\text {cool }}$ is calculated from the assumed balance between subsidence and radiative cooling

$$
w_{\mathrm{cool}} \frac{\partial \theta}{\partial z}=Q_{\mathrm{cool}},
$$

where $\partial \theta / \partial z$ is set to its pressure-weighted mean value in the layer $z=1.5-5 \mathrm{~km}$ (i.e., directly above the boundary layer) in the environmental sounding (see section $2 \mathrm{~h}$ ). For the control run, this gives $w_{\text {cool }}=0.25 \mathrm{~cm} \mathrm{~s}^{-1}$, which agrees well with the value of $0.24 \mathrm{~cm} \mathrm{~s}^{-1}$ obtained by calculating the mean (negative) vertical velocity in the region $r=[400$, $600] \mathrm{km}$ and $z=[1.5,5] \mathrm{km}$ from the equilibrium state of the control simulation. Details of the application of this analytical model are reprised in a later section.

We define $V_{m}$ as the time mean of its 2-day-runningmean value over the equilibrium period to account for shifts in $r_{m}$ that smooth out $V_{m}$; equilibrium values of the two size variables, $r_{\mathrm{ew}}$ and $r_{0}$, are calculated directly from the final equilibrium radial profile.

\section{f. Experimental approach: Parametric sensitivities and dimensional analysis}

We perform a wide range of experiments in which we independently and systematically vary from control each of the eight external dimensional parameters that are potentially relevant to the dynamics of the system: $T_{\mathrm{sst}}, T_{\mathrm{tpp}}, Q_{\mathrm{cool}}, u_{\mathrm{sfc}}, l_{h}, l_{v}, f$, and $r_{0_{q}}$. For each of $l_{h}, l_{v}, f$, and $r_{0_{q}}$, we run simulations successively halving and doubling from the control value, while for the four thermodynamic parameters we run simulations each varying one parameter from control as follows: $T_{\mathrm{sst}}=$ $285,287.5,290,292.5,295,300,305,310 \mathrm{~K} ; T_{\text {tpp }}=238$, $225,213,200,188,175,163,150 \mathrm{~K} ; u_{\mathrm{sfc}}=10,5,4,3,2,1$, $0.5 \mathrm{~m} \mathrm{~s}^{-1}$; and $Q_{\text {cool }}=0.25,0.375,0.5,0.75,1,1.5,2,3$, $4 \mathrm{~K} \mathrm{day}^{-1}$. These ranges, listed in order of increasing potential intensity $V_{p}$, span a reasonable range of values of $V_{p}$ from 50 to $150 \mathrm{~m} \mathrm{~s}^{-1}$.

Some important modifications are made to accommodate the wide range of simulations presented here. The domain height is increased by $5 \mathrm{~km}$ in cases where the troposphere is deeper than control to ensure that the upper-level damping layer lies sufficiently far above the tropopause. For the above sensitivity experiments in which the equilibrium radius of maximum wind is less than the control value, the simulation is rerun at doubled horizontal resolution (i.e., $d x=2 \mathrm{~km}, L_{\text {domain }}=$ $6144 \mathrm{~km}$ ) to ensure that the inner storm core is comparably resolved. Last, the time step is halved for simulations in which the CFL condition is violated (four cases with $l_{h}=12000 \mathrm{~m}$; one case with $l_{h}=6000 \mathrm{~m}, T_{\mathrm{tpp}}=$ $250 \mathrm{~K}$, and $f=20 \times 10^{-5} \mathrm{~s}^{-1}$ ). 
The final scaling results indicate to which dimensional variables the equilibrium storm structure is systematically sensitive. Dimensional analysis is then applied to synthesize the results in a nondimensional framework.

\section{g. Potential intensity in $R C E$}

The architecture of this model RCE state enables the equation for the maximum potential intensity to be reformulated in a useful manner. The generalized potential intensity (Emanuel 2010) is given by

$$
V_{p}^{2}=\frac{C_{k}}{C_{d}} \frac{T_{\mathrm{sst}}-T_{\mathrm{tpp}}}{T_{\mathrm{tpp}}}\left(k_{0}^{*}-k\right) .
$$

Combining (7) with the surface enthalpy flux equation in (2) gives

$$
V_{p}^{2}=\frac{T_{\mathrm{sst}}-T_{\mathrm{tpp}}}{T_{\mathrm{tpp}}} \frac{F_{k}}{\rho C_{d}|\mathbf{u}|} .
$$

In RCE, column energy balance requires that the surface enthalpy flux into the column be exactly balanced by the column-integrated radiative cooling, which in this idealized setup is given by

$$
\begin{aligned}
F_{k} & =-\int_{p_{s}}^{0} C_{p} \frac{\partial T}{\partial t} \frac{d p}{g}=-\int_{p_{s}}^{0} C_{p} \frac{\partial \theta}{\partial t}\left(\frac{p}{p_{0}}\right)^{R_{d} / C_{p}} \frac{d p}{g} \\
& =C_{p} Q_{\mathrm{cool}} \frac{\overline{\Delta p}}{g},
\end{aligned}
$$

where $C_{p}$ is the specific heat of air; $\overline{\Delta p}$ given by

$\overline{\Delta p}=\frac{p_{0}}{1+R_{d} / C_{p}}\left[\left(\frac{p_{s}}{p_{0}}\right)^{1+R_{d} / C_{p}}-\left(\frac{p_{\mathrm{tpp}}}{p_{0}}\right)^{1+R_{d} / C_{p}}\right]$

is the mean pressure depth of the troposphere, reduced slightly by the adiabatic expansion term in the integrand of (9); and we have ignored any small contribution from Newtonian relaxation in the stratosphere. Substituting (9) into (8) results in

$$
V_{p}^{2}=\frac{T_{\mathrm{sst}}-T_{\mathrm{tpp}}}{T_{\mathrm{tpp}}} \frac{C_{p} Q_{\mathrm{cool}} \overline{\Delta p}}{g \rho C_{d}|\mathbf{u}|} .
$$

Thus, it is readily apparent from (11) that the potential intensity in RCE with constant tropospheric cooling is a function of four externally defined parameters: $T_{\text {sst }}$, $T_{\mathrm{tpp}}, u_{\mathrm{sfc}}$, and $Q_{\text {cool }}$, with $\overline{\Delta p}$ primarily a function of $T_{\mathrm{tpp}}$. This analytical result will be leveraged below, though all values of potential intensity presented herein are calculated from the environmental sounding beyond the storm within the solution itself (see subsequent section) using the detailed Emanuel subroutine (Bister and Emanuel 2002) with zero boundary layer wind speed reduction under pseudoadiabatic thermodynamics and including dissipative heating. This follows from the work of Bryan and Rotunno (2009a), which identified the pseudoadiabatic version of potential intensity theory as the optimal choice; for the control simulation, the potential intensity under reversible physics is $7 \%$ less than for the pseudoadiabatic case. Although our model setup is not fully pseudoadiabatic, Bryan and Rotunno (2009b) found under simplified microphysics that storm intensity is not very sensitive to terminal fall speed except near the reversible limit.

\section{$h$. Defining the environmental sounding}

Though we initialize each axisymmetric simulation with the three-dimensional RCE state, ultimately the more relevant environmental sounding for the equilibrium tropical cyclone is that of the ambient environment beyond the storm circulation in the axisymmetric model solution itself. Thus, we define the environmental sounding as the area-weighted mean vertical profile of potential temperature and water vapor averaged over the radial grid points 2000-2500, which corresponds to the region $r=[8000,10000] \mathrm{km}$ for our control domain size. This quantity is largely insensitive to radius or averaging time period so long as it is calculated beyond the primary storm circulation. From this environmental sounding, we calculate relevant quantities for our analysis, including the potential intensity, radiative-subsidence rate, and deformation radius.

The potential intensity for the axisymmetric state is typically $80 \%-90 \%$ of the value of the corresponding three-dimensional RCE state, though they do not differ precisely by a constant factor across simulations. As in the three-dimensional case, the axisymmetric $V_{p}$ is predominantly a function of the four governing thermodynamic parameters. This quantity does not vary significantly with $l_{h}, f$, or domain size. This fact suggests that the difference in $V_{p}$ between the 3D environment without a storm and the 2D (axisymmetric) environment that includes a storm is related not to the existence of the storm itself but rather to the differing nature of convection, and its aggregate effect on the mean state, in $2 \mathrm{D}$ geometry as compared to $3 \mathrm{D}$. This is curious and warrants further investigation.

\section{Results}

\section{a. Control run}

Figure 3 displays the time evolution of the 2-day running mean of $V_{m}, r_{m}$, and $r_{0}$ for the control simulation as 


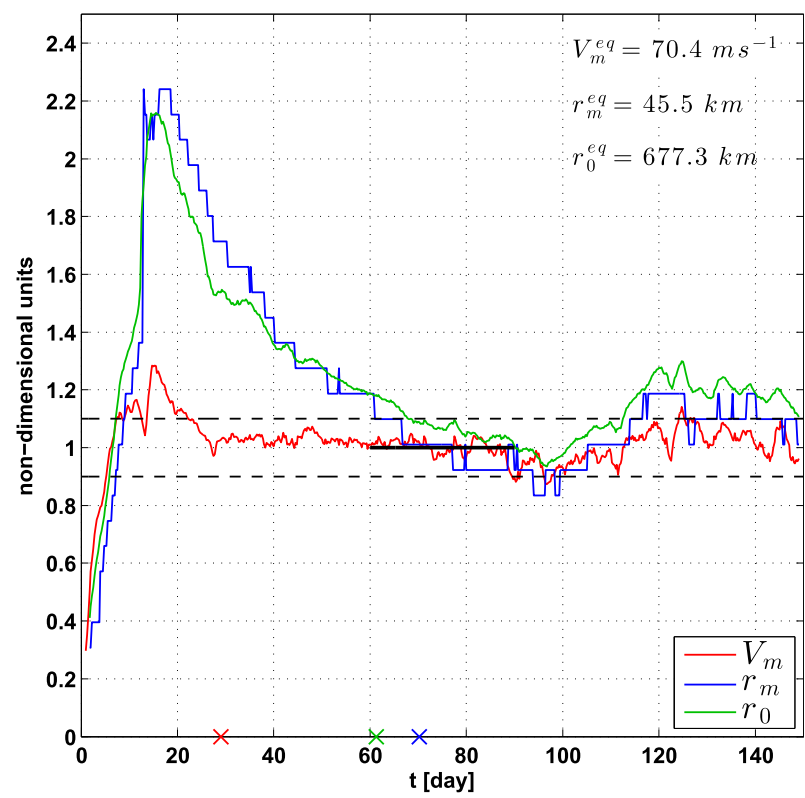

FIG. 3. For the control simulation, time evolution of the 2-dayrunning-mean $V_{m}, r_{m}$, and $r_{0}$ normalized by their respective equilibrium values (upper-right corner). For this simulation, $V_{p}=79 \mathrm{~m} \mathrm{~s}^{-1}$ and $f=5 \times 10^{-5} \mathrm{~s}^{-1}$. Black line denotes 30-day period used for equilibrium calculation, and black dashed lines denote $\pm 10 \%$ of the equilibrium value. Markers along the abscissa denote estimated time scales to equilibration.

well as estimated time scales to equilibrium for each individual variable. The time scale to equilibrium $\tau_{x}$ where $x$ is the variable of interest, is defined as the starting time of the first 30-day interval whose mean value is within $10 \%$ of the equilibrium value and whose average daily rate of change over this period does not exceed $1 \%$ of the mean. All three variables exhibit similar qualitative evolutions: rapid increase during genesis to a superequilibrium value followed by a more gradual decay to equilibrium. However, the maximum excess over equilibrium is very large for $r_{0}$ and $r_{m}(\sim 100 \%)$ and relatively small $(\sim 30 \%)$ for $V_{m}$, the latter of which matches that found in Hakim (2011), though this result is not considered fundamental, as the degree of overshoot may depend on model and environmental parameters. Moreover, the time scales to equilibrium are significantly longer for size $\left(\tau_{r_{m}}=70\right.$ days and $\tau_{r_{0}}=61$ days $)$ than for intensity $\left(\tau_{V}=\right.$ 29 days). The details of the transient phase of the structural evolution will be explored in future work.

These results suggest that modeling tropical cyclones over a period sufficient to achieve quasi equilibrium in intensity (typically 10-20 days), as is commonly done in the literature, may result in a storm that has not reached structural equilibrium or else has done so artificially because of the domain limitation imposed by the model's outer wall.

\section{b. Radial profile sensitivity tests}

The principal objective is to collapse the radial wind profiles across all simulations to a single curve based on external parameters alone. Thus, we begin simply with the dimensional radial gradient wind profiles for eight simulations sets, each of which correspond to one of the eight external dimensional parameters, displayed in Fig. 4. First, both storm intensity and inner-core size (e.g., $\left.r_{m}\right)$ increase with increasing potential intensity across all four thermodynamic parameters (Fig. 4, top two rows). Second, storm size decreases with increasing $f$ and increases with increasing $l_{h}$-the latter primarily only within the inner core-while storm intensity decreases with increasing $f$ and $l_{h}$ (Fig. 4, third row). Detailed analysis of the effects of the radial mixing length is found in Bryan and Rotunno (2009b) and Rotunno and Bryan (2012). Third, the equilibrium storm forgets the initial condition $r_{0_{q}}$ (Fig. 4, bottom right), as the small variability is not systematic in nature, with an identical result for an initial midlevel vortex (not shown; see Fig. 6 for scalings). Finally, storm intensity and overall size are not systematically sensitive to the vertical mixing length (Fig. 4, bottom left), which corroborates the results of Bryan and Rotunno (2009b) and Rotunno and Bryan (2012); larger vertical mixing length magnitudes do correspond to a slow expansion of the eye at the apparent expense of the eyewall, though its overall effect remains small relative to that of $l_{h}$, so long as $l_{v}$ is much smaller than the depth of the troposphere, as is easily the case for the range of plausible values. A much deeper discussion of the role of $l_{v}$ in the boundary layer in the broader context of classical vortex flow solutions with frictional boundary layers is discussed in Rotunno and Bryan (2012). Thus, based upon these results, we hereafter elect to neglect the effects of both the initial condition and the vertical mixing length, leaving only six external dimensional parameters.

Given the structural similarity apparent in the dimensional curves in Fig. 4, we propose to normalize $V$ by $V_{m}$ and $r$ by $r_{\text {ew }}$; the result is shown in Fig. 5. Remarkably, this single normalization removes a large majority of the variability in each case, indicating that the dominant scales are the internal variables $V_{m}$ and $r_{\text {ew }}$, which we seek to relate to our external dimensional parameters.

Based on (11) and the common scaling of both intensity and size with $V_{p}$, we hypothesize that the primary role of the dimensional parameters $T_{\mathrm{sst}}, T_{\mathrm{tpp}}, Q_{\mathrm{cool}}$, and $u_{\mathrm{sfc}}$ is to modulate $V_{p}$. From among the four thermodynamic external parameters, the tropopause temperature is the simplest theoretically, such that its variability should affect only the potential intensity and the depth 

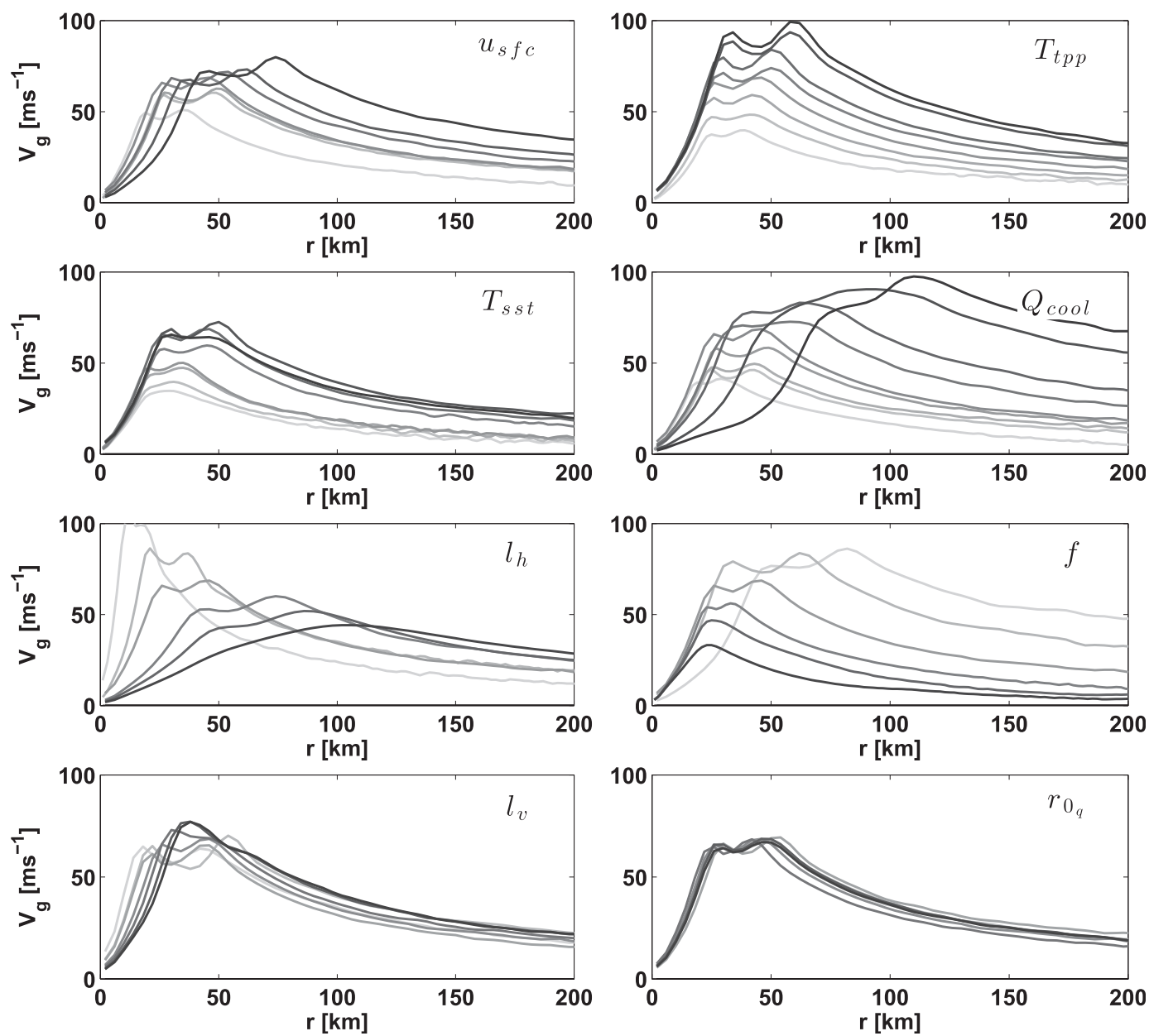

FIG. 4. Equilibrium radial profiles of the gradient wind for simulation sets in which each of the eight dimensional external parameters is varied. (top two rows) The four thermodynamic parameters, for which shading reflects potential intensity from low (light gray) to high (black); (bottom two rows) relevant dynamic parameters, for which shading reflects parameter magnitude from low (light gray) to high (black).

of the troposphere $H$. It will also slightly modulate the column-integrated radiative cooling, but because of the exponential decay in density with height, the mass of the troposphere varies by $\$ 15 \%$ over the range of tropopause temperatures explored here. Given that $H$ is not expected to be relevant to the dynamics of the system so long as $l_{v} / H \ll 1$ as noted earlier, we argue that $T_{\text {tpp }}$ represents the "base" case that isolates the variability in storm structure owing strictly to variations in $V_{p}$. We focus first on this base case $V_{p}\left(T_{\mathrm{tpp}}\right)$ before proceeding to analysis of the other three parameters, which may have additional effects on the system superimposed upon that associated with $V_{p}$.

\section{c. Base case: Inner core}

Figure 6 displays the scaling of $V_{m}$ and $r_{\text {ew }}$ with the set of relevant input physical parameters. Both structural variables exhibit systematic sensitivity to three parameters- $V_{p}\left(T_{\text {tpp }}\right)$, $f$, and $l_{h}$-with minimal sensitivity to the other parameters as noted above.

Rather than analyzing the role of each parameter independently though, we may synthesize the results quantitatively via dimensional analysis according to the Buckingham-Pi theorem. We have three relevant dimensional parameters and two fundamental measuresdistance and time - thereby giving only one independent nondimensional parameter: $C_{1}$. Any output nondimensional quantity $Y$ can be expressed as a function of $C_{1}$ :

$$
Y=f\left(C_{1}\right)
$$

The form of this functional relationship can only be determined by experimentation.

Thus, we define the dominant nondimensional number in this system as 

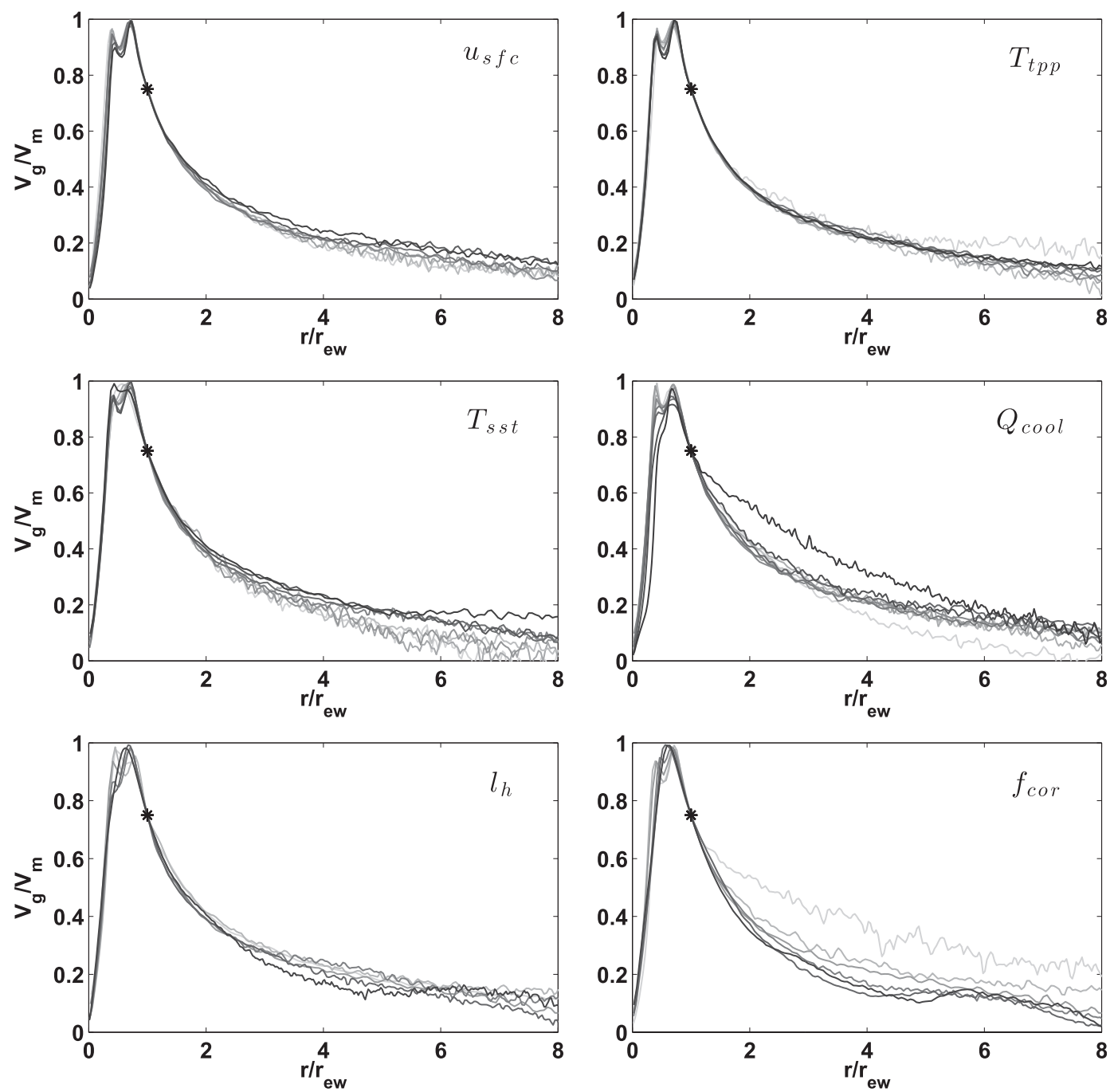

FIG. 5. As in Fig. 4, but with radial profiles normalized as follows: $V$ by $V_{m}$ and $r$ by $r_{\text {ew. }}$ Only those six parameters exhibiting strong structural sensitivity are shown.

$$
C_{1}=\frac{V_{p}}{f l_{h}}
$$

We choose to nondimensionalize $V_{m}$ by $V_{p}$ and $r_{\text {ew }}$ by $V_{p} / f$.

The scalings between the two nondimensional structural variables and $C_{1}$ for a suite of experiments varying one or more of $V_{p}, f$, or $l_{h}$ are displayed in Fig. 7; parameters for the set of experiments are given in Table 2. A linear relation in log-log space corresponds to a powerlaw scaling whose exponent is given by the linear slope; that is,

$$
Y=C_{1}^{\alpha}
$$

The linearly regressed slopes are also given in Fig. 7. In the case of $r_{\text {ew }}$, the power law indeed provides the best statistical fit. In the case of $V_{m}$ though, the $\log -\log$ plot exhibits slight negative curvature, particularly toward low values of $C_{1}$, indicating that a logarithmic relationship, $Y \sim \beta \log _{10}\left(C_{1}\right)$, provides a slightly better fit; this regression with $\beta=0.37$ is plotted as well (dasheddotted line). Though statistically slightly less precise, the power-law relationship is much more amenable to theoretical physical insight. The resulting nondimensional power-law relationships are given by

$$
\begin{gathered}
\frac{V_{m}}{V_{p}} \sim\left(\frac{V_{p}}{f l_{h}}\right)^{0.27}, \\
\frac{r_{\mathrm{ew}}}{V_{p} / f} \sim\left(\frac{V_{p}}{f l_{h}}\right)^{-0.55} .
\end{gathered}
$$



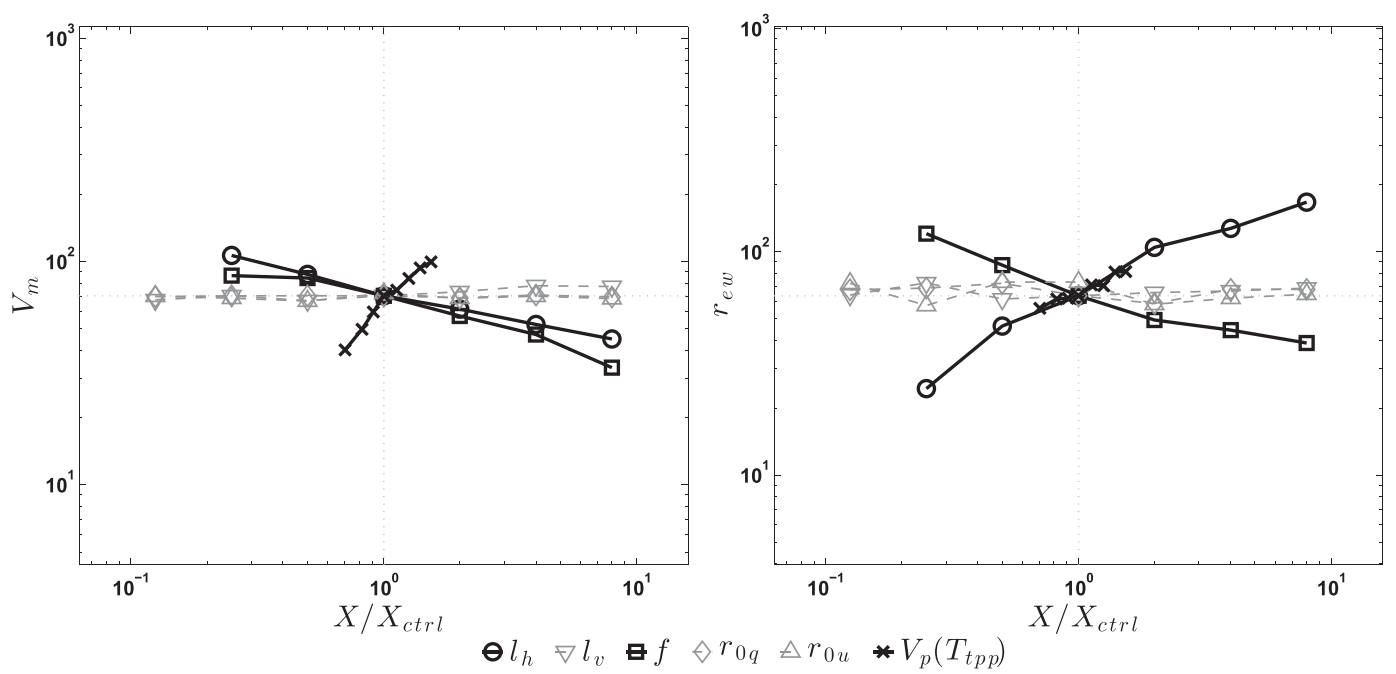

FIG. 6. Scaling of the equilibrium value of (left) $V_{m}$ and (right) $r_{\text {ew }}$ with relevant dimensional parameters $X$, normalized by their respective control values (abscissa). Parameters to which a structural variable exhibits systematic sensitivity are plotted in solid black.

We may then solve (15) for the corresponding dimensional scalings:

$$
\begin{aligned}
& V_{m} \sim V_{p}^{1.27}\left(f l_{h}\right)^{-0.27}, \\
& r_{m} \sim\left(\frac{V_{p}}{f}\right)^{0.45}\left(l_{h}\right)^{0.55} .
\end{aligned}
$$

Thus, equilibrium storm intensity is found to scale superlinearly with the potential intensity and, more weakly, inversely with both the background rotation rate and the radial turbulent mixing length. The equilibrium $r_{\mathrm{ew}}$, which scales closely with the radius of maximum gradient wind, is found to scale approximately as the geometric mean of the ratio of the potential intensity to the Coriolis parameter and the radial turbulent mixing length, weighted slightly toward the latter. Note that the direct nondimensional scaling for $r_{m}$ has an exponent of $\alpha=0.52$ and $r_{\text {adj }}^{2}=0.84$-both statistically indistinguishable from $r_{\text {ew }}$ at the $95 \%$ confidence level.

Curiously, the power dissipation (Emanuel 2005) follows the scaling

$$
\mathrm{PD} \sim V_{m}^{3} r_{m}^{2} \sim V_{p}^{4.7} f^{-1.7} l_{h}^{0.3}
$$

which exhibits only a very weak dependence on $l_{h}$.

\section{d. Base case: Outer radius}

We may now quantify the scaling of the overall storm size. We reiterate that $r_{0}$ is difficult to extract directly from numerical model output and thus elect to use the analytical outer wind model of Emanuel (2004) to represent the outer circulation. Following the above nondimensional scaling results, we first nondimensionalize $V$ by $V_{p}$ and $r$ by $V_{p} / f$ in (5), giving

$$
\frac{\partial(\tilde{r} \tilde{V})}{\partial \tilde{r}}=\frac{C_{d} V_{p}}{w_{\text {cool }}} \frac{2 \tilde{r}^{2} \tilde{V}^{2}}{\left(\tilde{r}_{0}^{2}-\tilde{r}^{2}\right)}-\tilde{r},
$$

where tildes denote nondimensional quantities. Chavas and Emanuel (2010) employed this model to estimate $r_{0}$ in observations by fitting the model to the radius of $12 \mathrm{~m} \mathrm{~s}^{-1}$. Here we find that (18) can credibly reproduce the entire equilibrium radial wind profile outside of the eyewall for many simulations with a simple empirical modification of the first term on the RHS of (18) by a constant factor $c=0.3$. As an example, Fig. 8 depicts the control simulation equilibrium profile compared against (18) fit without and with this modification (i.e., $c=1$ and $c=0.3$, respectively); see the supplemental material for analysis and discussion. To estimate $r_{0}$, we begin at $r_{\mathrm{ew}}=$ $r\left(0.75 V_{m}\right)$ from the equilibrium radial wind profile and integrate (18), with the first term on the RHS multiplied by $c=0.3$, outward to $r_{0}$. Because (18) reproduces the radial wind profile beyond the eyewall even as $r$ approaches $r_{m}$ for most simulations, the results are not sensitive to the precise radius used. A deeper understanding of this empirical fit is an important subject of future work. Note in Fig. 8 that the azimuthal winds reverse sign beyond $r_{0}$. Given that the flow at large radii beyond the TC has a large moment arm and also occupies a large cylindrical area, the area-integrated surface torque acting on a weak anticyclonic flow beyond the TC is sufficient to balance 


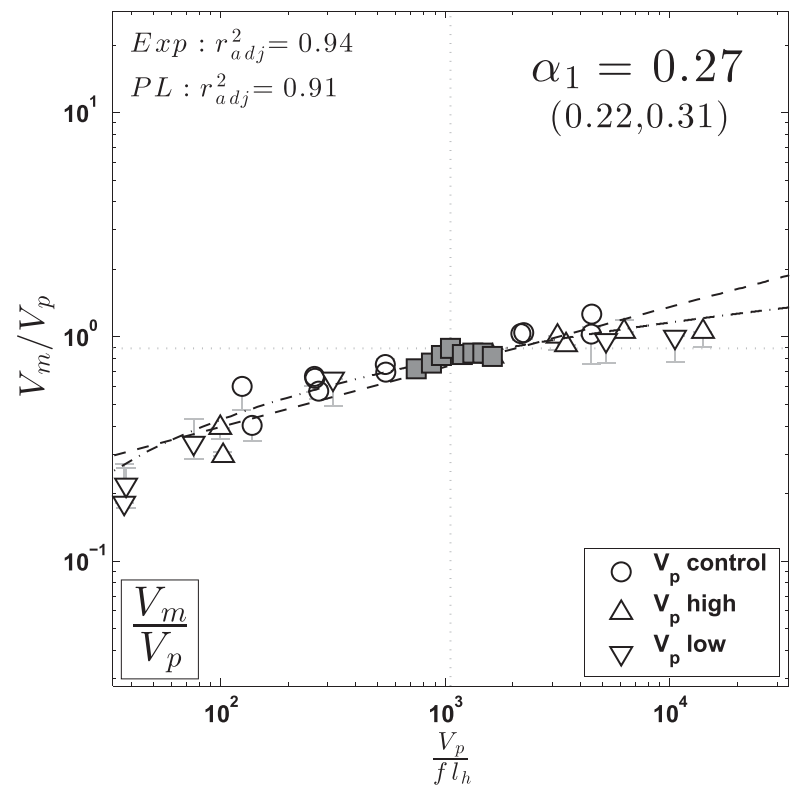

TABLE 2. Parameter values for each simulation used to test the scaling relationships associated with (12). The nondimensional parameter is $C_{1}=V_{p} /\left(f l_{h}\right)$. Control values are listed in Table 1 .

\begin{tabular}{|c|c|c|c|}
\hline$f\left(\times 10^{-5} \mathrm{~s}^{-1}\right)$ & $l_{h}(\mathrm{~m})$ & $V_{p}\left(T_{\mathrm{tpp}}\right)\left(\mathrm{m} \mathrm{s}^{-1}\right)$ & $C_{1}$ \\
\hline 10 & 12000 & 119 & 99 \\
\hline 20 & 6000 & 123 & 102 \\
\hline 10 & 12000 & 45 & 37 \\
\hline 5 & 12000 & 45 & 76 \\
\hline 1.25 & 750 & 132 & 14037 \\
\hline 20 & 6000 & 45 & 38 \\
\hline 1.25 & 750 & 49 & 5204 \\
\hline 1.25 & 375 & 49 & 10537 \\
\hline 2.5 & 750 & 117 & 6260 \\
\hline 2.5 & 1500 & 119 & 3164 \\
\hline 5 & 750 & 129 & 3453 \\
\hline 10 & 3000 & 79 & 263 \\
\hline 5 & 3000 & 47 & 316 \\
\hline 5 & 1500 & 79 & 1054 \\
\hline 5 & 1500 & 56 & 742 \\
\hline 5 & 1500 & 65 & 865 \\
\hline 5 & 1500 & 72 & 958 \\
\hline 5 & 1500 & 89 & 1189 \\
\hline 5 & 1500 & 99 & 1324 \\
\hline 5 & 1500 & 110 & 1471 \\
\hline 5 & 1500 & 122 & 1620 \\
\hline 1.25 & 1500 & 84 & 4473 \\
\hline 2.5 & 1500 & 82 & 2174 \\
\hline 10 & 1500 & 81 & 543 \\
\hline 20 & 1500 & 82 & 273 \\
\hline 40 & 1500 & 83 & 138 \\
\hline 5 & 375 & 84 & 4478 \\
\hline 5 & 750 & 84 & 2235 \\
\hline 5 & 3000 & 81 & 540 \\
\hline 5 & 6000 & 78 & 261 \\
\hline 5 & 12000 & 75 & 124 \\
\hline
\end{tabular}

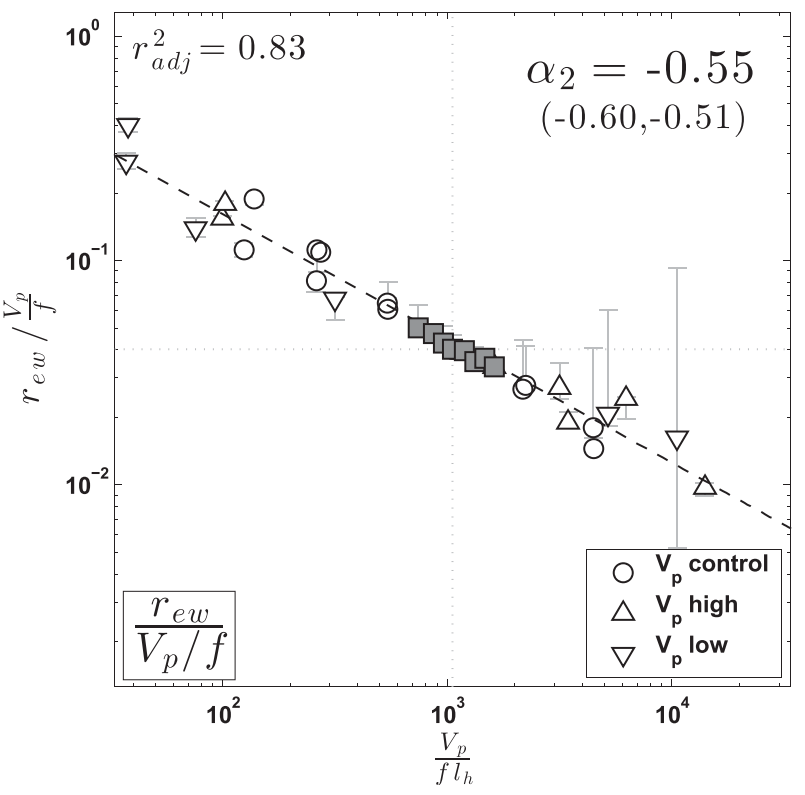

FIG. 7. Scaling of the equilibrium values of the nondimensionalized structural variable (top) $V_{m}$ and (bottom) $r_{\text {ew }}$ with the nondimensional number $C=V_{p} /\left(f l_{h}\right)$. Best-fit linear regressions are plotted (dashed), whose linearly regressed slopes correspond to the estimated power-law scaling exponent in (14), and associated $95 \%$ confidence intervals listed (parentheses) and $r$-squared values adjusted to account for the number of estimators (top-left corner). For $V_{m}$, a logarithmic regression is also shown (dashed-dotted). Gray filled highlights those simulations for which $V_{p}\left(T_{\text {tpp }}\right)$ alone is modulated. Gray bars indicate the full range of variability of the 30 -day running mean after day 60.

that acting on the TC cyclonic flow. As a simple estimate of surface torque balance, taking our control simulation equilibrium radial wind profile and assuming a homogeneous boundary layer yields the result that the net surface torque integrated over $r \leq r_{\tau}$ vanishes at approximately $r_{\tau}=2400 \mathrm{~km}$.

The top panel of Fig. 9 displays outer radial wind profiles for varying $T_{\text {tpp }}$, normalized as in Fig. 5. Overlaid on these radial profiles are the solutions of (18), each of which provides an estimate of $r_{0}$ (blue dots). The scaling of $r_{0}$ with $V_{p}$ is shown as an inset. The analytical model credibly reproduces the expansion of the normalized outer wind field with increasing $V_{p}$.

Indeed, (18) is itself modulated by a second nondimensional parameter, given by

$$
C_{2}=\frac{C_{d} V_{p}}{w_{\text {cool }}}
$$

We may quantify the impact of $C_{2}$ by simply holding it fixed at its control value (47.7) when solving (18); the result is shown in the bottom panel of Fig. 9. Comparison of the red curves in the top and bottom panels of Fig. 9 reveals that the effect of $C_{2}$ manifests itself primarily only 


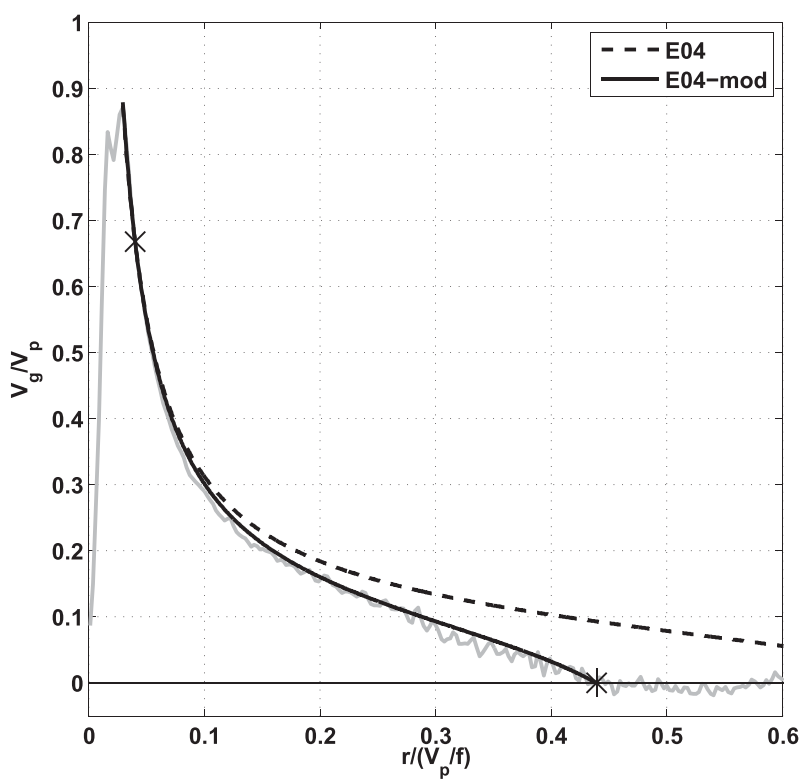

FIG. 8. Comparison of equilibrium radial wind profile for control simulation (gray) with (18) without (dashed) and with (solid) constant modification $(c=0.3)$, fit to $r\left(0.75 V_{m}\right)$ (marked with a cross).

at large radii in the far outer region of the storm circulation. Additionally, these new curves provide an estimate of an adjusted outer radius $r_{0}^{*}$ (blue dots, bottom panel), which is analogous to $r_{0}$, but with $C_{2}$ fixed at its control value. The scaling of $r_{0}^{*}$ with $V_{p}$ is shown as an inset. Physically, fixing $C_{2}$ acts to partially collapse the curves in the far outer region.

Though the influence of $C_{2}$ is minimal at smaller radii where wind speeds are an appreciable fraction of the maximum value, it exerts a significant influence on the precise value of $r_{0}$. As a result, the true $r_{0}$ is a function of $C_{1}$ and $C_{2}$, both of which include variability with $V_{p}$. Figure 10 displays the joint scaling of $r_{0}$ with $C_{1}$ and $C_{2}$ over a wide range of values of each. The values of $r_{0}$ are calculated beginning with the empirically derived relationships for $V_{m} / V_{p}$ (exponential) and $r_{\mathrm{ew}} /\left(V_{p} / f\right)$ (power law) as a function of $C_{1}$ displayed in Fig. 7 and given by

$$
\begin{aligned}
\frac{V_{m}}{V_{p}} & =-0.3+0.37 \log _{10}\left(C_{1}\right), \\
\frac{r_{\mathrm{ew}}}{V_{p} / f} & =0.73 C_{1}^{-0.55} .
\end{aligned}
$$

Then, for each $C_{1},(18)$ is applied to the corresponding $\left[r_{\mathrm{ew}} /\left(V_{p}\right) / f, V_{m} / V_{p}\right]$ using a range of values of $C_{2}$. In this way, we exploit the fact that the direct impacts of $C_{1}$ and $C_{2}$ are effectively independent in radius, with the former modulating the inner core of the storm and the latter
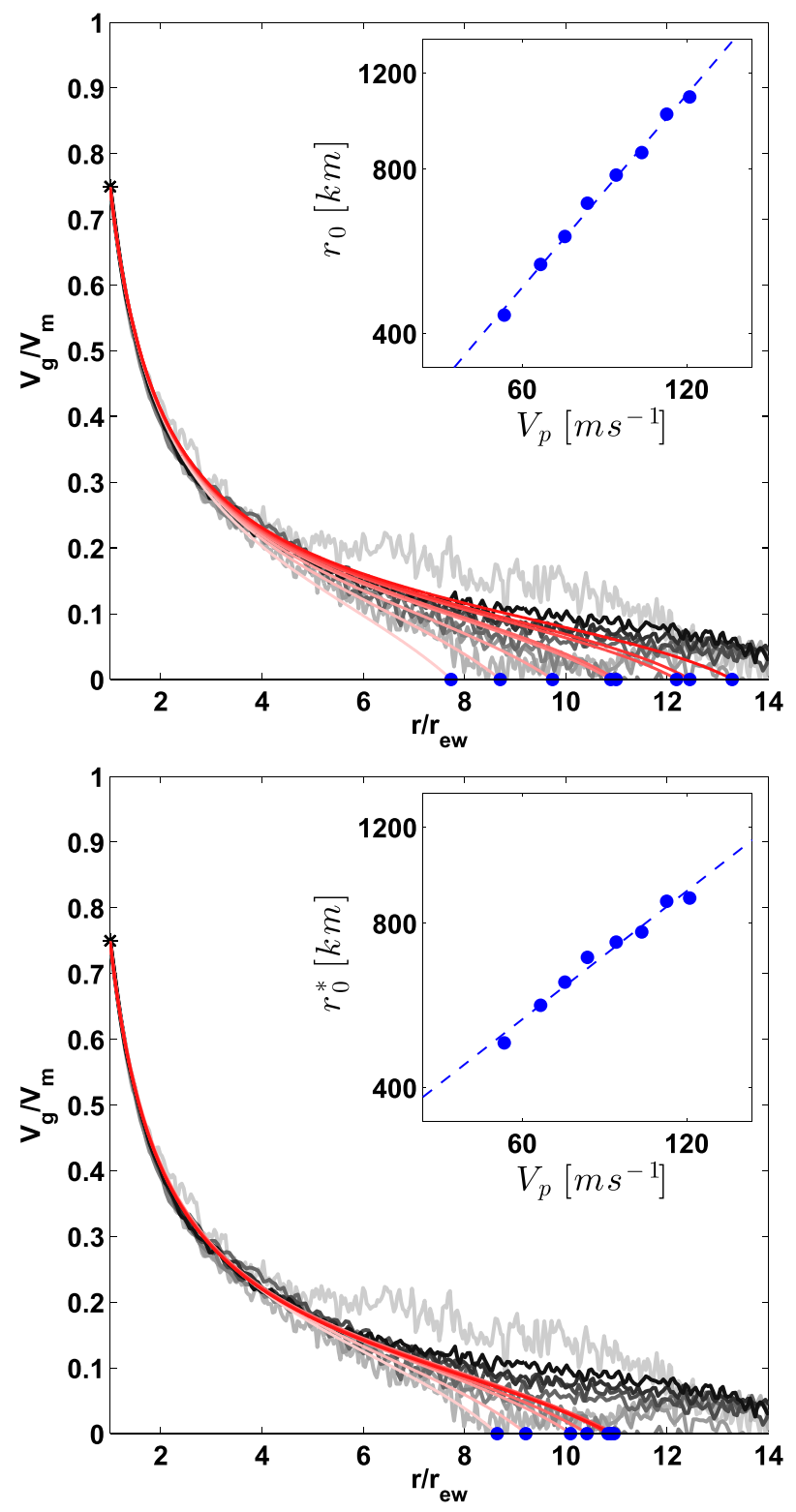

FIG. 9. As in Fig. 5 for varying $T_{\text {tpp }}$, except focused on the outer region of the storm. Black curves are the equilibrium radial wind profiles; red curves are solutions of (18) (top) fit directly and (bottom) fit with $C_{2}$ held fixed at its control value. Blue dots indicate corresponding (top) $r_{0}$ and (bottom) $r_{0}^{*}$, and the respective scalings with $V_{p}$ are shown as insets.

modulating the outer circulation; $r_{\mathrm{ew}}$ lies in the transition region between the two.

To quantify the variation of nondimensional $r_{0}$ with $C_{1}$ and $C_{2}$, a simple estimate of the separable power-law scaling can be obtained using multiple linear regressions. The result is given by

$$
\frac{r_{0}}{V_{p} / f} \sim C_{1}^{-0.2} C_{2}^{0.33}
$$




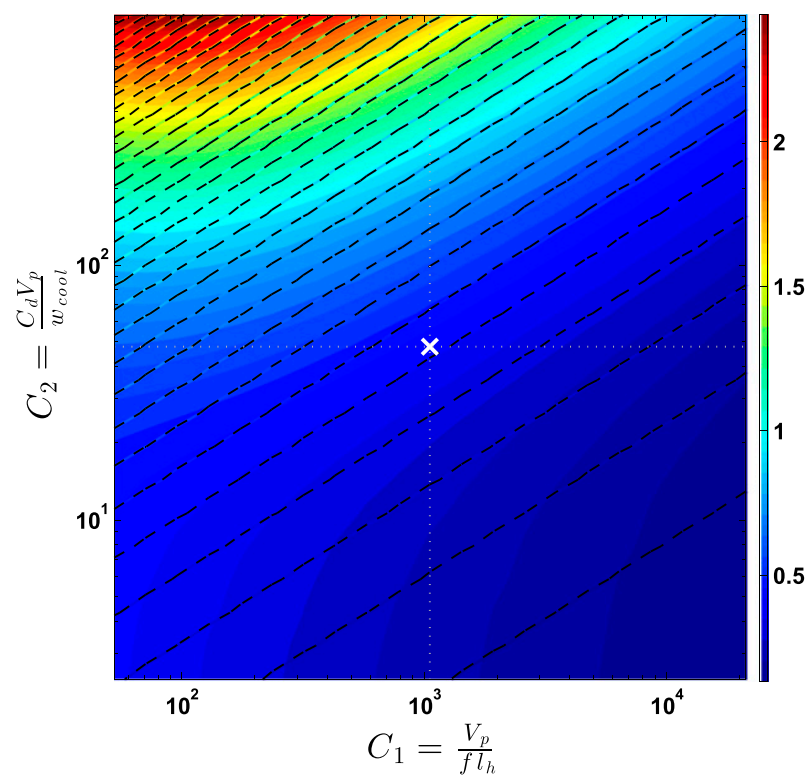

FIG. 10. Scaling of $r_{0} /\left(V_{p} / f\right)$ with $C_{1}$ and $C_{2}$, calculated using (18) with empirical fit to simulation results. White cross denotes control simulation. Contours depict power-law scaling fit given by (21).

and is plotted (contours) in Fig. 10. This statistical fit performs reasonably well except in regions of significant curvature (e.g., for small $C_{1}$ and large $C_{2}$ ). Notably, there is minimal curvature in the neighborhood of the control simulation.

The scaling of the nondimensional outer radius with $C_{1}$ while holding $C_{2}$ fixed at its control value, which corresponds to $r_{0}^{*}$ in Fig. 9, is equivalent to the application of dimensional analysis to $r_{0}^{*}$ as was done above for $V_{m}$ and $r_{\text {ew }}$. This exercise gives an exponent of -0.15 (not shown; $r_{\text {adj }}^{2}=0.97$ ), similar to the result from multiple linear regression. The corresponding dimensional scaling is

$$
r_{0}^{*} \sim\left(\frac{V_{p}}{f}\right)^{0.85}\left(l_{h}\right)^{0.15} .
$$

Thus, (22) dictates that, at fixed $C_{2}$, overall storm size is found to scale nearly linearly with the ratio of the potential intensity to the Coriolis parameter, with a slight expansion for increasing radial turbulent mixing length. This scaling matches the existing axisymmetric theoretical prediction for the scaling of the upper bound on the size of a tropical cyclone (Emanuel 1986, 1989, 1995a). This natural length scale is $\sqrt{\chi_{s}} / f$, where $\sqrt{\chi_{s}}$ is a velocity scale that is equivalent to the potential intensity with $C_{k}=C_{d}$ and neglecting dissipative heating and the pressure dependence on the saturation vapor pressure of water. From a Carnot heat engine perspective, the upper bound on storm size exists because the work required to restore lost angular momentum in the anticyclone aloft increases with increasing storm size, and by conservation of energy, there remains less energy available to overcome frictional dissipation at the surface (i.e., a weaker storm). To the extent that the inclusion of the pressure dependence of saturation vapor pressure and dissipative heating do not alter this fundamental principle, our modeling results appear to confirm this prediction.

Importantly, in order to isolate this theoretical scaling in a dimensionally consistent manner, one must first control for this secondary expansion associated with $C_{2}$, as we have done in calculating $r_{0}^{*}$; this seems reasonable given that the theory is applicable only to the ascending region of the storm and so should not be expected to represent variability in the nonconvecting outer circulation. Moreover, the result is very similar when applying (18) beginning at $r\left(0.2 V_{m}\right)$ (scaling exponent of -0.11 ), indicating that this result is not an artifact of the analytical outer wind model.

\section{e. Physical interpretation}

More generally, $C_{1}$ represents the ratio of the storm radial length scale $V_{p} / f$ to the parameterized eddy radial length scale $l_{h}$, and thus it is the values of each of these parameters relative to one another, rather than their absolute values, that is fundamental to the structure of the storm. For example, though one would expect $V_{m}$ to scale linearly with $V_{p}$ all else equal, the superlinearity is a manifestation of the fact that a larger value of $V_{p}$ results in a storm that is more intense and larger. A larger storm at constant $l_{h}$ implies a reduction in $C_{1}$, and thus the storm will feel a weaker effective turbulence. Indeed, from (14) for constant $C_{1}$ we do indeed recover the linear scaling $V_{m} \sim V_{p}$.

In addition, these findings corroborate prior work demonstrating the importance of radial turbulence in determining inner-core storm structure (Bryan and Rotunno 2009a; Bryan 2012; Rotunno and Bryan 2012). In particular, the strong scaling relationship between $r_{m}$ and $l_{h}$ reflects the critical role of radial turbulence in counteracting eyewall frontogenesis by the secondary circulation that, in the inviscid limit, would lead to frontal collapse (Emanuel 1997). Meanwhile, the influence of radial turbulence as parameterized here only weakly modifies the overall size of the storm.

The term $C_{2}$ represents the reciprocal of the nondimensional Ekman suction rate in the outer wind region, where the requirement that $w_{\mathrm{Ek}}=w_{\mathrm{cool}}$ has been imposed. Decreasing the Ekman suction rate implies through Ekman dynamics a weaker (negative) vorticity and thus a more gradual decay of the radial wind profile. In nondimensional space, the nondimensional suction 
rate can be decreased either by decreasing $w_{\text {cool }}$, which by assumption implies a smaller dimensional $w_{\mathrm{Ek}}$, or by increasing the scaling factor $C_{d} V_{p}$. In this way, $C_{2}$ governs the rate of decay of the wind profile with radius in the nondimensional system.

\section{f. Estimating $l_{h}$}

An accurate estimation of $l_{h}$ in the inner core of a real tropical cyclone is important but lacks any theoretical or observational foundation, as it is not a function of physically calculable natural variables. Bryan and Rotunno (2009b) and Bryan (2012) tune it to match the steady-state model intensity to either the theoretical potential intensity or the theoretical maximum gradient wind speed of Emanuel and Rotunno (2011). The above results suggest that the more relevant objective is to tune the ratio $V_{m} / V_{p}$ to the horizontal mixing length nondimensionalized by the storm scale $V_{p} / f$ (i.e., the reciprocal of $C_{1}$ ). We thus estimate this parameter value as that which gives the theoretical result from Emanuel and Rotunno (2011) of $V_{m} / V_{p}=1 / \sqrt{2}$ in the case of $C_{k} / C_{d}=1$, calculated using the logarithmic scaling for $V_{m} / V_{p}$ (Fig. 7, top). The resulting best estimate is $l_{h} /\left(V_{p} / f\right)=0.0017$, or approximately $1 / 600$ of the storm radial length scale. For our control values for $V_{p}$ and $f$, this result translates to $l_{h} \approx 2700 \mathrm{~m}$. This value seems reasonable in the context of previous work that finds optimal values in the range $1000-1500 \mathrm{~m}$ given that those simulations were performed in domains approximately half the size required to avoid influencing storm size (Fig. 2). This result disagrees with an observationally based estimate of $l_{h}=700 \mathrm{~m}$ (Zhang and Montgomery 2012), though without a suitable storm scale with which to normalize this value, the two results are not directly comparable. Moreover, here we are focused on the equilibrium storm, whereas real-world storms are likely far from equilibrated.

\section{g. Sensitivity to potential intensity}

We now return to the hypothesis that the sensitivity of storm structure to each of the four thermodynamic parameters collapses to a sensitivity to potential intensity. Figure 11 displays the respective scalings of $V_{m}, r_{\mathrm{ew}}$, and $r_{0}^{*}$ with $V_{p}$. Indeed, across all four parameters there is a systematic, direct scaling with $V_{p}$ in both intensity and size, with several interesting deviations. Implicitly, any variability above and beyond the scaling with $V_{p}\left(T_{\mathrm{tpp}}\right)$ is necessarily a result of modulation of some other aspect of the system that is correlated with $V_{p}$. For $V_{m}$, the slightly superlinear scaling with $V_{p}$ matches that found for $T_{\text {tpp }}$ in all cases. For $r_{e w}$, the scaling with $V_{p}$ for both $u_{\text {sfc }}$ and $Q_{\text {cool }}$ is faster than for $T_{\text {tpp }}$, and the scaling diverges nonlinearly at very high radiative cooling rates.
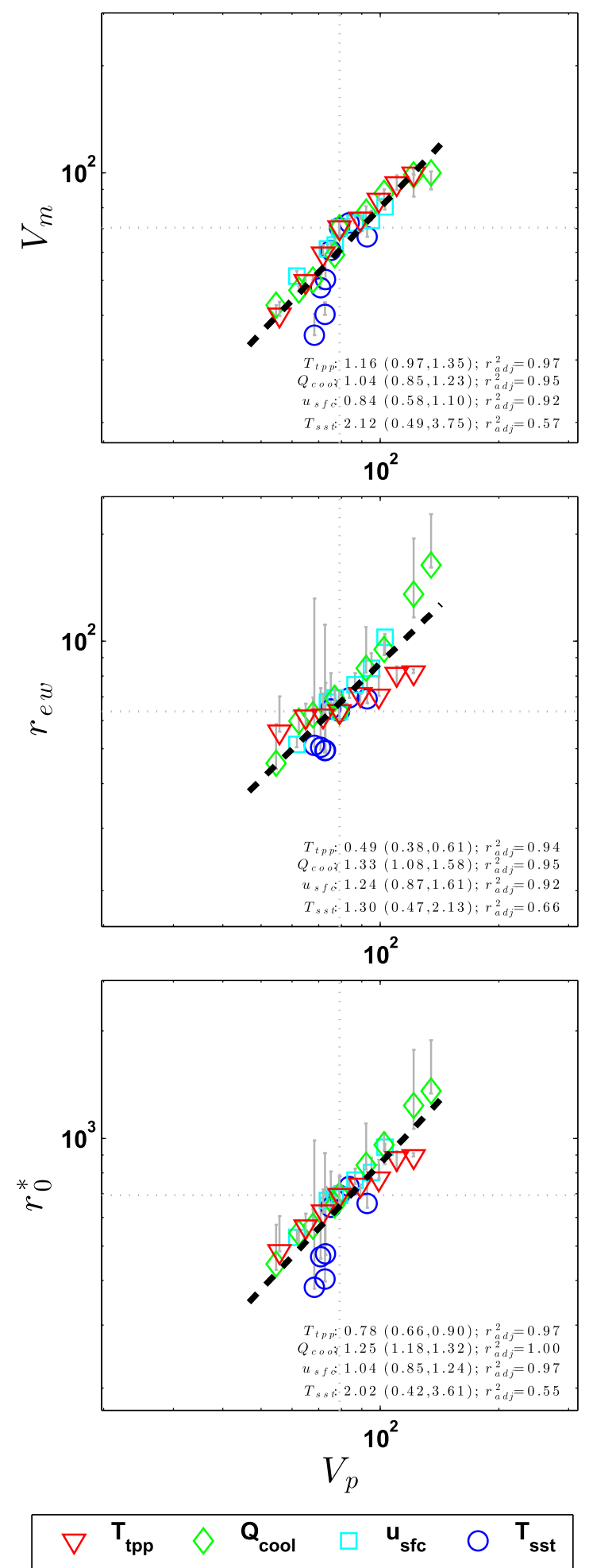

FIG. 11. Scaling of the respective equilibrium values of $V_{m}, r_{\mathrm{ew}}$, and $r_{0}^{*}$ (ordinate) with $V_{p}$ (abscissa) for the four thermodynamic parameters. Dashed line indicates best fit to all data. For each individual parameter, best-fit linear slope (95\% confidence interval) and corresponding adjusted $r$-squared statistic listed in lower-right corner. Gray bars indicate full range of variability of the 30-day running mean after day 60 . 
Finally, for $r_{0}^{*}$, the scalings largely collapse with the exception of high radiative cooling rates as was seen for $r_{\text {ew }}$. The two cases with coldest $T_{\text {sst }}(285$ and $287.5 \mathrm{~K})$ do not conform well to the overall scaling but instead are weaker and smaller than expected given their potential intensities, though these simulations exhibit significant ongoing variability during the postequilibration period. Results are very similar when applying (18) beginning at $r\left(0.2 V_{m}\right)$, reflecting the fact that (18) does a reasonable job representing the radial wind profile radially inwards of $r\left(0.2 V_{m}\right)$.

Deviations in $r_{\mathrm{ew}}$ may be attributable to the behavior of the eye. As seen in Fig. 4, the radial wind profile in the eye expands outward as $u_{\text {sfc }}$ is decreased and as $Q_{\text {cool }}$ is increased; this expansion is positively correlated with the expansion associated with increasing $V_{p}$. In particular, storm size expands at high radiative cooling rates, perhaps because of the development of significant convection beyond the eyewall, which may cause an expansion of the wind field (Hill and Lackmann 2009). It should be noted though, that the variability in storm structure increases significantly at these more extreme conditions. For the largest radiative cooling rate $\left(4 \mathrm{~K} \mathrm{day}^{-1}\right)$, the storm develops wind maxima at radii around $1000 \mathrm{~km}$ before eventually developing a more traditional tropical radial wind structure only around day 120 . More generally, this behavior may reflect the fact that in radiative-convective equilibrium, precipitation must balance free-tropospheric radiative cooling. Thus, total precipitation necessarily must increase with $Q_{\text {cool }}$, but given constraints on the terminal velocity of raindrops, as well as the decrease in boundary layer water vapor mixing ratio with increasing $Q_{\text {cool }}$, this implies that the areal coverage of precipitation must increase, which may result in a larger storm. At sufficiently high values of $Q_{\text {cool }}$, a precipitation distribution that is confined to the eyewall region, as it appears to be in typical axisymmetric simulations, may no longer be sufficient to balance radiative cooling, and thus convection will necessarily develop at larger radii. A more detailed analysis of this hypothesis is beyond the scope of this work.

\section{h. Rossby deformation radius}

One potentially relevant length scale from conventional geostrophic adjustment theory that has not been discussed to this point is the Rossby deformation radius, defined as

$$
L_{R}=\frac{N_{v} H}{f}
$$

where $N_{v}^{2}=\left(g / \theta_{v}\right)\left(\partial \theta_{v} / \partial z\right)$ is the buoyancy frequency and $H$ is the fluid depth (Emanuel 1994, p. 166). One

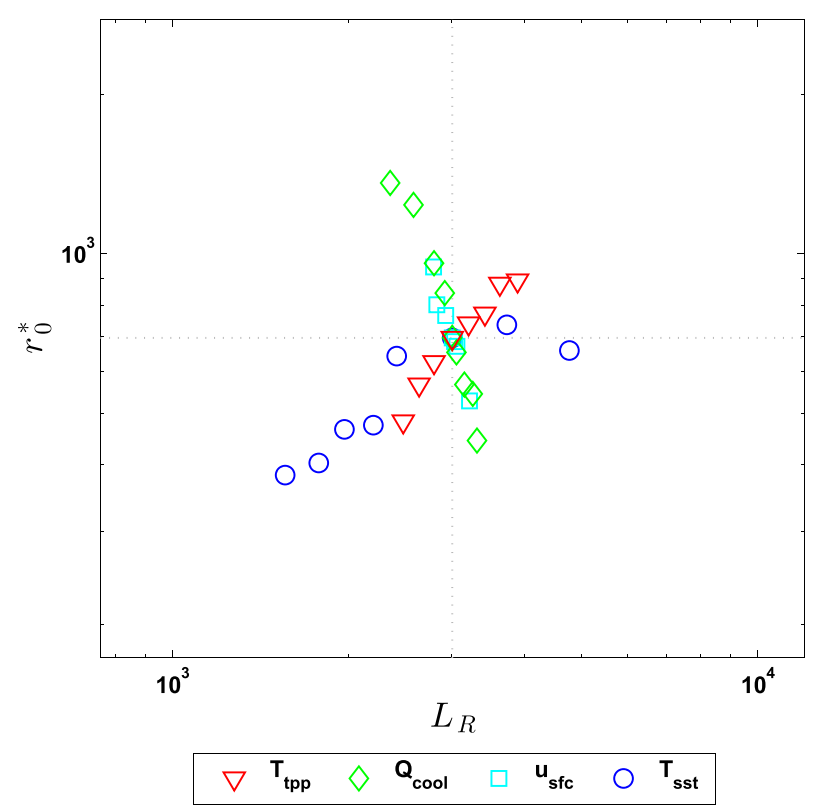

FIG. 12. As in Fig. 11, but for the scaling of $r_{0}^{*}$ with the Rossby deformation radius.

plausible explanation for the finding that the relevant storm length scale is $V_{p} / f$ is that this quantity simply covaries with the deformation radius. Indeed, in a threedimensional rotating radiative-convective equilibrium simulation, Held and Zhao (2008) noted a scaling for the size of their tropical cyclone that was consistent with either $L_{R}$ or $V_{p} / f$ but could not distinguish between the two based on the given parameter space. Here we test this hypothesis in Fig. 12, which is analogous to the bottom panel of Fig. 11, but for the scaling of $r_{0}^{*}$ with $L_{R}$ rather than $V_{p}$ (which is equivalent to $V_{p} / f$ since $f$ is fixed). The deformation radius is calculated from the vertical profiles of potential temperature and water vapor in the environmental sounding, where $H$ is the depth of the troposphere, taken to be the linearly interpolated altitude where the temperature first drops below $T_{\mathrm{tpp}}$, and $N_{v}$ is taken as the tropospheric pressure-weighted mean. In the case of varying $T_{\mathrm{sst}}$ and $T_{\mathrm{tpp}}, r_{0}^{*}$ indeed scales in the same direction for both $L_{R}$ and $V_{p}$. In contrast, the scaling of $r_{0}^{*}$ with $u_{\mathrm{sfc}}$ and $Q_{\text {cool }}$ is of the opposite sign. Taken together, Fig. 12 suggests that $L_{R}$ is not fundamental to the scaling of the equilibrium storm, noting that this conclusion applies equivalently to $V_{m}$ and $r_{m}$ given that both exhibit similar qualitative scaling behavior (i.e., positive and monotonic with $V_{p}$ ).

Physically, the distinct scaling relationship of these two parameters is the manifestation of their convenient effect on our idealized RCE state: an increase in $Q_{\text {cool }}$ and a decrease in $u_{\mathrm{sfc}}$ both act to increase the air-sea thermodynamic disequilibrium, $k_{s}^{*}-k$, which increases 
$V_{p}$ [see (11)], while simultaneously decreasing $N_{v}$ and $H$, which decreases $L_{R}$.

Why the deformation radius lacks relevance is not obvious. Past work analyzing the axisymmetric and weakly asymmetric balance dynamics of an evolving vortex forced by sources of heat and momentum (Eliassen 1952; Shapiro and Montgomery 1993; Vigh and Schubert 2009) found the deformation scale to be the fundamental horizontal storm scale. We hypothesize that the discrepancy with our results lies in the nature of the forcing: the aforementioned work imposed a localized external source of heat to force the circulation, while here the system is forced everywhere by radiative cooling, out of which emerges a tropical cyclone whose circulation and convective heating fields exist simultaneously without explicit cause-effect. In the former, the system is characterized by one of perpetual geostrophic adjustment to external heating whose governing scale is the deformation radius; in the latter, the forcing carries no intrinsic horizontal length scale and thus an alternative scale emerges, which we find is given by $V_{p} / f$. Moreover, direct application of a Sawyer-Eliassen calculation to the model heating fields, which would be expected to yield a deformation length scale, is not appropriate because it assumes that the return flow is adiabatic, whereas the return flow subsidence in a steady tropical cyclone is balanced by radiative cooling.

\section{Conclusions}

This work combines highly idealized modeling, motivated by existing axisymmetric tropical cyclone theory, with dimensional analysis to systematically quantify the scaling relationship between the structure of a model tropical cyclone at statistical equilibrium and relevant model, initial, and environmental external parameters. We perform this analysis in a model world whose complexity is reduced so as to retain only the essential physics of the tropical atmosphere necessary to produce a tropical cyclone: radiative-convective equilibrium in axisymmetric geometry on an $f$ plane with constant tropospheric cooling, constant background gustiness (to provide a background source of water vapor), constant surface exchange coefficients for momentum and enthalpy, and constant sea surface and tropopause temperatures. We find that storm structure at statistical equilibrium is primarily a function of only three parameters: the potential intensity, the Coriolis parameter, and the radial turbulent mixing length. These three parameters constitute the dominant nondimensional parameter $V_{p} / f l_{h}$ for the equilibrium system. This parameter can be interpreted as the ratio of the storm radial length scale $V_{p} / f$ to the radial eddy mixing scale $l_{h}$ and thus dictates the effective turbulence felt by a storm relative to its size - a finding that is perhaps unsurprising in the context of extant tropical cyclone theory that is itself phrased entirely in terms of relative rather than absolute radial length scales (Emanuel 1995b). A second nondimensional parameter $C_{d} V_{p} / w_{\text {cool }}$, whose reciprocal represents the nondimensional Ekman suction rate, exists within a preexisting slab boundary layer outer wind model that, given a simple empirical modification, can reproduce the outer wind field of a tropical cyclone across a range of simulations. We find that the overall size of the storm, at fixed nondimensional Ekman suction rate, scales nearly linearly with $V_{p} / f$, which is the theoretical scaling for the upper bound on tropical cyclone size derived in Emanuel (1986).

The extent to which these equilibrium results can be applied to real storms in nature is not clear for two key reasons. First, the time scales to equilibrium identified here for the control simulation are significantly longer than the lifespan of tropical cyclones on Earth. Second, storms in nature rarely exist in a truly quasi-steady environment for more than a couple of days, if at all. Indeed, the large range in the observed size distribution cannot be explained by the equilibrium results; Chavas and Emanuel (2010) noted that nondimensionalization by $V_{p} / f$ had little impact on their results. However, equilibrium dynamics may potentially manifest itself more clearly at an aggregate level, such that shifts in the global distribution of $V_{p} / f$ within the main tropical cyclone basins may translate into shifts in the size distribution of tropical cyclones, even though variability within the global distribution is the result of more complex nonequilibrium processes. For example, global warming due to a doubling of atmospheric carbon dioxide concentrations is expected to lead to a global increase in potential intensity of approximately 10\% (Emanuel 1987; Knutson et al. 2010). Much more work is needed to assess the extent to which such a relationship is borne out in models while accounting for shifts in the spatial distribution of potential intensity, as well as tropical cyclone genesis locations and tracks. Nonetheless, gaining an understanding of the dynamics of the equilibrium tropical cyclone in an idealized environment is the first step toward an understanding of the full evolution of tropical cyclones in nature.

Opportunities for future work abound. Sensitivity to the surface exchange coefficients, as well as the transient evolution of size and structure, are currently under investigation. Exploration of the analytical outer wind field boundary layer model and its application to the nonconvecting outer region of a tropical cyclone is needed to understand both the physics of our empirical modification and the validity of the analytical model when applied to a simulation with a more properly resolved boundary layer. Deeper analysis of the deviations from the uniform 
scaling with potential intensity, such as within the eye, may elucidate the physics behind additional modes of variability specific to each governing thermodynamic parameter. Moreover, there may be other modes of variability in storm structure in the figures presented herein that went unnoticed but that, to the keen eye, warrant further research. The impact of factors that limit storm intensity, such as midlevel ventilation (Tang and Emanuel 2010), on storm size and structure remains unexplored. Three-dimensional simulations, in which turbulence is more properly resolved, may be explored to test the validity of the axisymmetric results. Finally, application and extension of this work to real-world tropical cyclones remains an open question, including the more complicated time-dependent dynamics associated with the transient phase of storm evolution in our idealized modeling environment. Such a fundamental physical understanding would ideally translate into a capacity for credible prediction of storm size, structure, and evolution at the level of individual storms, as well as insight into how the distribution of storm size may differ in other climate states. Both would be beneficial for the purposes of emergency preparedness and risk management alike.

Acknowledgments. Thanks to Greg Hakim, Marty Singh, and Tim Cronin for a number of very useful discussions in the course of this work. Many thanks to George Bryan (NCAR) for providing public access to his model, CM1. This research is supported by NSF Grant 1032244, as well as the Department of Energy Office of Science Graduate Fellowship Program (DOE SCGF), made possible in part by the American Recovery and Reinvestment Act of 2009, administered by ORISEORAU under Contract DE-AC05-06OR23100.

\section{REFERENCES}

Bister, M., and K. A. Emanuel, 1997: The genesis of Hurricane Guillermo: TEXMEX analyses and a modeling study. Mon. Wea. Rev., 125, 2662-2682, doi:10.1175/1520-0493(1997)125<2662: TGOHGT $>2.0 . \mathrm{CO} ; 2$.

_ , and K. Emanuel, 2002: Low frequency variability of tropical cyclone potential intensity. Part 1: Interannual to interdecadal variability. J. Geophys. Res., 107, 4801, doi:10.1029/ 2001JD000776.

Bretherton, C. S., P. N. Blossey, and M. Khairoutdinov, 2005: An energy-balance analysis of deep convective self-aggregation above uniform SST. J. Atmos. Sci., 62, 4273-4292, doi:10.1175/ JAS3614.1.

Bryan, G. H., 2012: Effects of surface exchange coefficients and turbulence length scales on the intensity and structure of numerically simulated hurricanes. Mon. Wea. Rev., 140, 11251143, doi:10.1175/MWR-D-11-00231.1.

_ , and J. M. Fritsch, 2002: A benchmark simulation for moist nonhydrostatic numerical models. Mon. Wea. Rev.,
130, 2917-2928, doi:10.1175/1520-0493(2002)130<2917: ABSFMN $>2.0 . \mathrm{CO} ; 2$.

, and R. Rotunno, 2009a: Evaluation of an analytical model for the maximum intensity of tropical cyclones. J. Atmos. Sci., 66, 3042-3060, doi:10.1175/2009JAS3038.1.

— , and — 2009b: The maximum intensity of tropical cyclones in axisymmetric numerical model simulations. Mon. Wea. Rev., 137, 1770-1789, doi:10.1175/2008MWR2709.1.

Chavas, D. R., and K. A. Emanuel, 2010: A QuikSCAT climatology of tropical cyclone size. Geophys. Res. Lett., 37, L18816, doi:10.1029/2010GL044558.

DeMaria, M., and J. D. Pickle, 1988: A simplified system of equations for simulation of tropical cyclones. J. Atmos. Sci., 45, 1542-1554, doi:10.1175/1520-0469(1988)045<1542:ASSOEF>2.0.CO;2.

Eliassen, A., 1952: Slow thermally or frictionally controlled meridional circulation in a circular vortex. Astrophys. Norv., 5 (2), $19-60$.

Emanuel, K. A., 1986: An air-sea interaction theory for tropical cyclones. Part I: Steady-state maintenance. J. Atmos. Sci., 43, 585605, doi:10.1175/1520-0469(1986)043<0585:AASITF>2.0.CO;2. 1987: The dependence of hurricane intensity on climate. Nature, 326, 483-485, doi:10.1038/326483a0.

1989: The finite-amplitude nature of tropical cyclogenesis. J. Atmos. Sci., 46, 3431-3456, doi:10.1175/ 1520-0469(1989)046<3431:TFANOT >2.0.CO;2.

1994: Atmospheric Convection. Oxford University Press, 580 pp. [Available online at http://books.google.com/books? $\mathrm{id}=$ VdaBBHEGAcMC. $]$

_ 1995a: The behavior of a simple hurricane model using a convective scheme based on subcloud-layer entropy equilibrium. J. Atmos. Sci., 52, 3960-3968, doi:10.1175/ 1520-0469(1995)052<3960:TBOASH > 2.0.CO;2.

1995b: Sensitivity of tropical cyclones to surface exchange coefficients and a revised steady-state model incorporating eye dynamics. J. Atmos. Sci., 52, 3969-3976, doi:10.1175/ 1520-0469(1995)052<3969:SOTCTS >2.0.CO;2.

, 1997: Some aspects of hurricane inner-core dynamics and energetics. J. Atmos. Sci., 54, 1014-1026, doi:10.1175/ 1520-0469(1997)054<1014:SAOHIC >2.0.CO;2.

, 2004: Tropical cyclone energetics and structure. Atmospheric Turbulence and Mesoscale Meteorology, E. Fedorovich, R. Rotunno, and B. Stevens, Eds., Cambridge University Press, 165-192.

- 2005: Increasing destructiveness of tropical cyclones over the past 30 years. Nature, 436, 686-688, doi:10.1038/nature03906.

- 2010: Tropical cyclone activity downscaled from NOAACIRES reanalysis, 1908-1958. J. Adv. Model. Earth Syst., 2 (1), doi:10.3894/JAMES.2010.2.1.

_ , and R. Rotunno, 2011: Self-stratification of tropical cyclone outflow. Part I: Implications for storm structure. J. Atmos. Sci., 68, 2236-2249, doi:10.1175/JAS-D-10-05024.1.

Frank, W. M., 1977: The structure and energetics of the tropical cyclone. Part I: Storm structure. Mon. Wea. Rev., 105, 1119-1135, doi:10.1175/1520-0493(1977)105<1119:TSAEOT>2.0.CO;2.

Hakim, G. J., 2011: The mean state of axisymmetric hurricanes in statistical equilibrium. J. Atmos. Sci., 68, 1364-1386, doi:10.1175/ 2010JAS3644.1.

Hartmann, D. L., J. R. Holton, and Q. Fu, 2001: The heat balance of the tropical tropopause, cirrus, and stratospheric dehydration. Geophys. Res. Lett., 28, 1969-1972, doi:10.1029/2000GL012833.

Held, I. M., and M. Zhao, 2008: Horizontally homogeneous rotating radiative-convective equilibria at GCM resolution. J. Atmos. Sci., 65, 2003-2013, doi:10.1175/2007JAS2604.1. 
Hill, K. A., and G. M. Lackmann, 2009: Influence of environmental humidity on tropical cyclone size. Mon. Wea. Rev., 137, 3294 3315, doi:10.1175/2009MWR2679.1.

Iman, R. L., M. E. Johnson, and C. C. Watson, Jr., 2005: Sensitivity analysis for computer model projections of hurricane losses. Risk Anal., 25, 1277-1297, doi:10.1111/j.1539-6924.2005.00673.x.

Irish, J. L., D. T. Resio, and J. J. Ratcliff, 2008: The influence of storm size on hurricane surge. J. Phys. Oceanogr., 38, 2003 2013, doi:10.1175/2008JPO3727.1.

Knutson, T. R., and Coauthors, 2010: Tropical cyclones and climate change. Nat. Geosci., 3, 157-163, doi:10.1038/ngeo779.

Lee, C.-S., K. K. W. Cheung, W.-T.. Fang, and R. L. Elsberry, 2010: Initial maintenance of tropical cyclone size in the western North Pacific. Mon. Wea. Rev., 138, 3207-3223, doi:10.1175/ 2010MWR3023.1.

Lin, Y.-L., R. D. Farley, and H. D. Orville, 1983: Bulk parameterization of the snow field in a cloud model. J. Climate Appl. Meteor., 22, 1065-1092, doi:10.1175/1520-0450(1983)022<1065: $\mathrm{BPOTSF}>2.0 . \mathrm{CO} ; 2$.

Merrill, R. T., 1984: A comparison of large and small tropical cyclones. Mon. Wea. Rev., 112, 1408-1418, doi:10.1175/ 1520-0493(1984)112<1408:ACOLAS > 2.0.CO;2.

Nolan, D. S., E. D. Rappin, and K. A. Emanuel, 2007: Tropical cyclogenesis sensitivity to environmental parameters in radiative-convective equilibrium. Quart. J. Roy. Meteor. Soc., 133, 2085-2107, doi:10.1002/qj.170.

Ooyama, K., 1969: Numerical simulation of the life cycle of tropical cyclones. J. Atmos. Sci., 26, 3-40, doi:10.1175/ 1520-0469(1969)026<0003:NSOTLC $>2.0$. CO;2.

Powell, M. D., P. J. Vickery, and T. A. Reinhold, 2003: Reduced drag coefficient for high wind speeds in tropical cyclones. Nature, 422, 279-283, doi:10.1038/nature01481.

Rotunno, R., and K. A. Emanuel, 1987: An air-sea interaction theory for tropical cyclones. Part II: Evolutionary study using a nonhydrostatic axisymmetric numerical model. $J$. Atmos. Sci., 44, 542-561, doi:10.1175/1520-0469(1987)044<0542: AAITFT $>2.0 . \mathrm{CO} ; 2$.
_ , and G. H. Bryan, 2012: Effects of parameterized diffusion on simulated hurricanes. J. Atmos. Sci., 69, 2284-2299, doi:10.1175/JAS-D-11-0204.1.

Shapiro, L. J., and M. T. Montgomery, 1993: A threedimensional balance theory for rapidly rotating vortices. J. Atmos. Sci., 50, doi:10.1175/1520-0469(1993)050<3322: ATDBTF $>2.0 . \mathrm{CO} ; 2$.

Smagorinsky, J., 1963: General circulation experiments with the primitive equations. Mon. Wea. Rev., 91, 99-164, doi:10.1175/ 1520-0493(1963)091<0099:GCEWTP>2.3.CO;2.

Smith, R. K., and M. T. Montgomery, 2008: Balanced boundary layers used in hurricane models. Quart. J. Roy. Meteor. Soc., 134, 1385-1395, doi:10.1002/qj.296.

— , and S. Vogl, 2008: A simple model of the hurricane boundary layer revisited. Quart. J. Roy. Meteor. Soc., 134, 337-351, doi:10.1002/qj.216.

_ C. W. Schmidt, and M. T. Montgomery, 2011: An investigation of rotational influences on tropical-cyclone size and intensity. Quart. J. Roy. Meteor. Soc., 137, 1841-1855, doi:10.1002/qj.862.

Tang, B., and K. Emanuel, 2010: Midlevel ventilation's constraint on tropical cyclone intensity. J. Atmos. Sci., 67, 1817-1830, doi:10.1175/2010JAS3318.1.

Vigh, J. L., and W. H. Schubert, 2009: Rapid development of the tropical cyclone warm core. J. Atmos. Sci., 66, 3335-3350, doi:10.1175/2009JAS3092.1.

Weatherford, C., and W. Gray, 1988: Typhoon structure as revealed by aircraft reconnaissance. Part I: Data analysis and climatology. Mon. Wea. Rev., 116, 1032-1043, doi:10.1175/ 1520-0493(1988)116<1032:TSARBA > 2.0.CO;2.

$\mathrm{Xu}$, J., and Y. Wang, 2010: Sensitivity of the simulated tropical cyclone inner-core size to the initial vortex size. Mon. Wea. Rev., 138, 4135-4157, doi:10.1175/2010MWR3335.1.

Zhang, J. A., and M. T. Montgomery, 2012: Observational estimates of the horizontal eddy diffusivity and mixing length in the low-level region of intense hurricanes. J. Atmos. Sci., 69 , 1306-1316, doi:10.1175/JAS-D-11-0180.1. 